\title{
DESARROLLO DE UNA APLICACIÓN PARA IMPLEMENTAR UN MODELO PREDICTIVO DE INYECCIÓN QUÍMICA
}

\author{
DeVelopment of an application to implement a chemical flood \\ PREDICTIVE MODEL
}

Jorge Luis Ramos Ramos*

Natalia Alejandra Angarita Buitrago**

Solangie Viviana Buitrago Peña***

Recibido: 11 de enero de 2017

Aceptado: 25 de abril de 2017

\section{Resumen}

Se presenta una aplicación desarrollada en Visual Basic que permite determinar el comportamiento de campos petrolíferos donde se aplican procesos de inyección de agua, polímeros, surfactantes y surfactantes-polímeros, a través de un modelo analítico que considera la teoría de BuckleyLeverett y el método de tubos de corriente. La aplicación no reemplaza la simulación numérica, porque no tiene en cuenta el modelo estático del reservorio y, en general, requiere poca información de entrada; sin embargo, es una aproximación rápida de predicción para obtener tablas y gráficas de tasas de producción y acumulados de petróleo y agua y factor de recobro de petróleo y comparar los resultados con los obtenidos por otros tipos de herramientas predictivas.

Palabras clave: inyección química, modelo predictivo, recobro mejorado.

\section{Abstract}

It is presented an application developed in the Visual Basic programming language that allows to determine the behavior of oil fields, in which processes of waterflooding, polymer flooding, surfactant flooding and surfactant-polymer flooding are applied through an analytical model that considers the Buckley-Leverett theory and the streamtubes method. The application does not replace the numerical simulation because it does not consider the static model of the reservoir and, in general, it requires little input information. However, it is a quick approach of prediction that makes possible to obtain summary tables and graphs of oil and water production rates and cumulative production and oil recovery factor, besides comparing with results obtained from other type of predictive tools.

Keywords: chemical flooding, predictive model, enhanced oil recovery.

\footnotetext{
* Ingeniero de petróleos, magíster en Ingeniería. Docente investigador, grupo de investigación Simulación de Yacimientos y Recobro Mejorado del Petróleo, Fundación Universidad de América. jorge.ramos@profesores.uamerica.edu.co ** Estudiante coinvestigador, grupo de investigación Simulación de Yacimientos y Recobro Mejorado del Petróleo, Programa Ingeniería de Petróleos, Fundación Universidad de América.nata_ale19@hotmail.com

*** Estudiante coinvestigador, grupo de investigación Simulación de Yacimientos y Recobro Mejorado del Petróleo, Programa Ingeniería de Petróleos, Fundación Universidad de América. solabp09@hotmail.com
} 


\section{INTRODUCCIÓN}

La simulación numérica detallada es utilizada frecuentemente en la selección apropiada de métodos de recobro mejorado del petróleo (EOR, sigla en inglés de enhanced oil recovery), sin embargo, presenta inconvenientes que limitan su pertinencia; así, además de ser costosa, su realización y análisis de resultados requiere un tiempo prolongado, ya que no siempre está disponible toda la información necesaria del yacimiento.

Por esta razón, un pronóstico menos complejo es de gran ayuda. Desde hace algunos años, el estudio de situaciones ha permitido elaborar modelos predictivos (correlaciones numéricas que caracterizan los procesos) mediante análisis de sensibilidades para entender la influencia de diferentes variables que gobiernan el proceso de recobro y determinan su eficiencia.

En este sentido, es importante contar con herramientas o programas que realicen pronósticos utilizando estos modelos predictivos. Por ejemplo, el software EOR es una interfaz gráfica de usuario diseñada por Petroleum Solutions Ltd. (2010) para Estados Unidos, el Departamento de Energía de Estados Unidos (DOE) y el Laboratorio Nacional de Tecnología Energética que permite evaluar rápidamente yacimientos y cuantificar incremental de producción al aplicar técnicas EOR a partir de modelos desarrollados (entre 1982 y 1984) por el Consejo Nacional de Petróleo de Estados Unidos (NPC) y el DOE.

Con este fin, se diseñó una aplicación (software) que incluyó modelos predictivos para la inyección de agua, polímeros, surfactantes y surfactantes-polímeros, desarrollados por los autores de este artículo (ver Angarita, Buitrago y Ramos, 2016). Este estudio describe el método de tubos de corriente utilizado, la estructura general del programa, sus tres etapas de desarrollo: información mínima requerida, resultados y ajuste, y el funcionamiento.

\section{Materiales y métodos}

La aplicación Chemical Prediction (CHP) se codifica en Visual Basic, un lenguaje de programación desarrollado por Alan Cooper para Microsoft, y permite determinar el comportamiento de campos en los que se aplican procesos de inyección de agua, polímeros, surfactantes y surfactantespolímeros.

Este modelo predictivo considera el modelo para inyección de polímeros PFPM (polymer flood predictive model), creado para el Departamento de Energía de Estados Unidos. El modelo comprende una modificación del factor de corrección por efecto de tapón de polímero finito y tiene en cuenta más variables correlacionables que afectan el proceso de inyección; además, permite obtener un pronóstico con más tubos de corriente para incrementar la resolución de los cálculos de barrido areal, es decir, del área horizontal del yacimiento inundado.

De acuerdo a lo anterior, la herramienta predice el desempeño del reservorio para una inyección continua del químico, por medio de la teoría de flujo fraccional de Buckley-Leverett y el método de tubos de corriente; igualmente, con los ajustes generados por Angarita et ál. (2016), se calcula el factor de corrección por el tamaño del bache que se inyecta y se estima finalmente la producción. A continuación se describe el método de tubos de corriente utilizado en el modelo predictivo con los respectivos cambios.

\section{Tubos de corriente}

Teniendo en cuenta que los modelos predictivos buscan simplificar la metodología utilizada para pronosticar el esquema de desarrollo de un yacimiento, la aproximación conseguida es muy útil cuando se consideran tubos de corriente o canales de flujo. 
Higgins y Leighton (1962, citados por Willhite, 1986, pp. 120-128) dieron a conocer un método que ayuda a estimar el flujo de dos fases en reservorios complejos utilizando términos geométricos. Estos autores consideran que las partículas de fluido se mueven desde el pozo inyector hasta el pozo productor siguiendo caminos, a los que llaman líneas de corriente, y que no existe flujo a través de ellos; por lo tanto, se genera una región rodeada por dos líneas de corriente, denominada tubo o canal de corriente, que permite el movimiento de un volumen específico de fluido. El modelo asume que el proceso de desplazamiento inmiscible sigue las mismas líneas de corriente que fueron establecidas para estudiar el flujo estable de un fluido homogéneo en el medio poroso y que, además, cada tubo de corriente se divide en bloques de igual volumen (en este caso se trabaja con veinte).

En el modelo PFPM, cada cuadrante de cada capa del reservorio se divide en uno, cuatro u ocho tubos de corriente y se simula el desplazamiento inmiscible de fluidos (dentro de un tubo de corriente) mediante la teoría de Buckley-Leverett, al considerar que el frente de invasión avanza una celda por cada paso de tiempo hasta que ocurre la ruptura en la última celda. Igualmente, con el fin de pronosticar el comportamiento de todo el patrón de flujo, se suman los resultados en un paso de tiempo de todos los tubos de corriente y todas las capas que conforman la porción del reservorio en estudio. En ese caso, a partir de ocho tubos de corriente se obtiene el modelo para uno y cuatro tubos de corriente, con una relación de proporción sencilla; por ejemplo, al considerar cuatro tubos de corriente se suma la fracción areal de un bloque en el primer tubo de corriente con el correspondiente en el segundo tubo de corriente. La tabla 1 presenta los valores de la fracción areal de cada bloque en cada tubo de corriente, y la tabla 2, la fracción areal de cada tubo de corriente.

Tabla 1. Fracción areal de cada bloque en cada tubo de corriente para ocho tubos de corriente

\begin{tabular}{|c|c|c|c|c|c|c|c|c|c|}
\hline & & \multicolumn{8}{|c|}{ Número de tubos de corriente } \\
\hline & & 1 & 2 & 3 & 4 & 5 & 6 & 7 & 8 \\
\hline \multirow{20}{*}{ 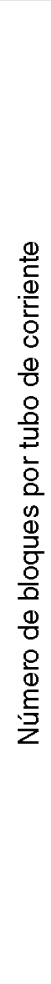 } & 1 & 0.0000005 & 0.0000005 & 0.0000005 & 0.0000005 & 0.0000005 & 0.0000005 & 0.0000005 & 0.0000005 \\
\hline & 2 & 0.0000023 & 0.0000023 & 0.0000023 & 0.0000023 & 0.0000023 & 0.0000023 & 0.0000023 & 0.0000023 \\
\hline & 3 & 0.0000084 & 0.0000084 & 0.0000084 & 0.0000084 & 0.0000084 & 0.0000084 & 0.0000084 & 0.0000084 \\
\hline & 4 & 0.0000301 & 0.0000301 & 0.0000301 & 0.0000301 & 0.0000301 & 0.0000301 & 0.0000301 & 0.0000301 \\
\hline & 5 & 0.0001063 & 0.0001063 & 0.0001063 & 0.0001063 & 0.0001063 & 0.0001063 & 0.0001063 & 0.0001063 \\
\hline & 6 & 0.0003740 & 0.0003740 & 0.0003740 & 0.0003740 & 0.0003740 & 0.0003740 & 0.0003740 & 0.0003740 \\
\hline & 7 & 0.0013148 & 0.0013148 & 0.0013147 & 0.0013146 & 0.0013145 & 0.0013144 & 0.0013143 & 0.0013143 \\
\hline & 8 & 0.0046310 & 0.0046292 & 0.0046259 & 0.0046216 & 0.0046169 & 0.0046127 & 0.0046094 & 0.0046077 \\
\hline & 9 & 0.0167663 & 0.0166744 & 0.0165121 & 0.0163135 & 0.0161135 & 0.0159409 & 0.0158159 & 0.0157506 \\
\hline & 10 & 0.1343376 & 0.0712345 & 0.0591854 & 0.0526867 & 0.0486082 & 0.0459724 & 0.0443689 & 0.0436063 \\
\hline & 11 & 0.2519089 & 0.1257947 & 0.1018587 & 0.0890600 & 0.0811029 & 0.0760038 & 0.0729218 & 0.0714620 \\
\hline & 12 & 0.2640441 & 0.1378399 & 0.1137449 & 0.1007519 & 0.0925995 & 0.0873320 & 0.0841283 & 0.0826050 \\
\hline & 13 & 0.2673603 & 0.1411543 & 0.1170561 & 0.1040589 & 0.0959019 & 0.0906303 & 0.0874234 & 0.0858984 \\
\hline & 14 & 0.2683011 & 0.1420951 & 0.1179968 & 0.1049995 & 0.0968424 & 0.0915707 & 0.0883638 & 0.0868386 \\
\hline & 15 & 0.2685688 & 0.1423628 & 0.1182645 & 0.1052672 & 0.0971101 & 0.0918384 & 0.0886314 & 0.0871063 \\
\hline & 16 & 0.2686450 & 0.1424390 & 0.1183407 & 0.1053434 & 0.0971863 & 0.0919146 & 0.0887076 & 0.0871825 \\
\hline & 17 & 0.2686667 & 0.1424606 & 0.1183624 & 0.1053651 & 0.0972080 & 0.0919363 & 0.0887293 & 0.0872042 \\
\hline & 18 & 0.2686729 & 0.1424668 & 0.1183685 & 0.1053712 & 0.0972142 & 0.0919424 & 0.0887355 & 0.0872104 \\
\hline & 19 & 0.2686746 & 0.1424686 & 0.1183703 & 0.1053730 & 0.0972159 & 0.0919442 & 0.0887372 & 0.0872121 \\
\hline & 20 & 0.2686751 & 0.1424691 & 0.1183708 & 0.1053735 & 0.0972164 & 0.0919447 & 0.0887377 & 0.0872126 \\
\hline
\end{tabular}

Nota. Adaptado de DOE y MEMV (1986b). 
JORGE RAMOS, NATALIA ANGARITA Y SOLANGIE BUITRAGO

Tabla 2. Fracción areal de cada tubo de corriente para ocho tubos de corriente

\begin{tabular}{cccccccc}
\hline \multicolumn{8}{c}{ Número de tubos de corriente } \\
\hline $\mathbf{1}$ & $\mathbf{2}$ & $\mathbf{3}$ & $\mathbf{4}$ & $\mathbf{5}$ & $\mathbf{6}$ & $\mathbf{7}$ & $\mathbf{8}$ \\
\hline 0.2686751 & 0.1424691 & 0.1183708 & 0.1053735 & 0.0972164 & 0.0919446 & 0.0887377 & 0.08721263 \\
\hline
\end{tabular}

Nota. Adaptado de DOE y MEMV (1986b)

De manera análoga, se generaron valores de fracción areal para cada bloque en dieciséis y veinticuatro tubos de corriente, destinados a la aplicación propuesta (Chemical Prediction-CHP); se divide la fracción areal de cada bloque en el modelo de ocho tubos de corriente, en dos y en tres tubos de corriente, respectivamente (teniendo en cuenta los valores de las tablas 1 y 2). En las tablas 3 y 4 se presentan los valores de la fracción areal de cada bloque en cada tubo de corriente, y en las tablas 5 y 6 , la fracción areal de cada tubo de corriente para 16 tubos de corriente.

Tabla 3. Fracción areal de cada bloque en cada tubo de corriente para 16 tubos de corriente (parte 1)

\begin{tabular}{|c|c|c|c|c|c|c|c|c|c|}
\hline & & \multicolumn{8}{|c|}{ Número de tubos de corriente } \\
\hline & & 1 & 2 & 3 & 4 & 5 & 6 & 7 & 8 \\
\hline \multirow{20}{*}{ 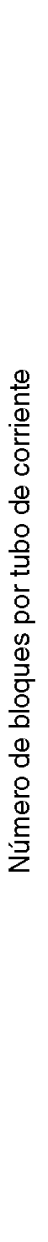 } & 1 & 0.0000003 & 0.0000003 & 0.0000003 & 0.0000003 & 0.0000003 & 0.0000003 & 0.0000003 & 0.0000003 \\
\hline & 2 & 0.0000011 & 0.0000011 & 0.0000011 & 0.0000011 & 0.0000011 & 0.0000011 & 0.0000011 & 0.0000011 \\
\hline & 3 & 0.0000042 & 0.0000042 & 0.0000042 & 0.0000042 & 0.0000042 & 0.0000042 & 0.0000042 & 0.0000042 \\
\hline & 4 & 0.0000151 & 0.0000151 & 0.0000151 & 0.0000151 & 0.0000150 & 0.0000150 & 0.0000151 & 0.0000151 \\
\hline & 5 & 0.0000531 & 0.0000532 & 0.0000531 & 0.0000532 & 0.0000531 & 0.0000533 & 0.0000532 & 0.0000531 \\
\hline & 6 & 0.0001870 & 0.0001870 & 0.0001870 & 0.0001870 & 0.0001870 & 0.0001870 & 0.0001870 & 0.0001870 \\
\hline & 7 & 0.0006574 & 0.0006574 & 0.0006574 & 0.0006574 & 0.0006573 & 0.0006573 & 0.0006573 & 0.0006573 \\
\hline & 8 & 0.0023155 & 0.0023155 & 0.0023146 & 0.0023146 & 0.0023129 & 0.0023129 & 0.0023108 & 0.0023108 \\
\hline & 9 & 0.0083831 & 0.0083831 & 0.0083372 & 0.0083372 & 0.0082560 & 0.0082560 & 0.0081567 & 0.0081567 \\
\hline & 10 & 0.0671688 & 0.0671688 & 0.0356173 & 0.0356173 & 0.0295927 & 0.0295927 & 0.0263434 & 0.0263434 \\
\hline & 11 & 0.1259544 & 0.1259544 & 0.0628974 & 0.0628974 & 0.0509293 & 0.0509293 & 0.0445300 & 0.0445300 \\
\hline & 12 & 0.1320221 & 0.1320220 & 0.0689199 & 0.0689199 & 0.0568724 & 0.0568725 & 0.0503760 & 0.0503759 \\
\hline & 13 & 0.1336801 & 0.1336801 & 0.0705771 & 0.0705771 & 0.0585280 & 0.0585280 & 0.0520294 & 0.0520294 \\
\hline & 14 & 0.1341506 & 0.1341506 & 0.0710475 & 0.0710475 & 0.0589984 & 0.0589984 & 0.0524997 & 0.0524997 \\
\hline & 15 & 0.1342844 & 0.1342844 & 0.0711814 & 0.0711814 & 0.0591323 & 0.0591323 & 0.0526336 & 0.0526336 \\
\hline & 16 & 0.1343225 & 0.1343225 & 0.0712195 & 0.0712195 & 0.0591703 & 0.0591703 & 0.0526717 & 0.0526717 \\
\hline & 17 & 0.1343333 & 0.1343333 & 0.0712303 & 0.0712303 & 0.0591812 & 0.0591812 & 0.0526825 & 0.0526825 \\
\hline & 18 & 0.1343364 & 0.1343364 & 0.0712334 & 0.0712334 & 0.0591842 & 0.0591843 & 0.0526856 & 0.0526856 \\
\hline & 19 & 0.1343373 & 0.1343373 & 0.0712343 & 0.0712343 & 0.0591851 & 0.0591851 & 0.0526865 & 0.0526865 \\
\hline & 20 & 0.1343376 & 0.1343376 & 0.0712345 & 0.0712345 & 0.0591854 & 0.0591854 & 0.0526867 & 0.0526867 \\
\hline
\end{tabular}


Tabla 4. Fracción areal de cada bloque en cada tubo de corriente para 16 tubos de corriente (parte 2)

\begin{tabular}{|c|c|c|c|c|c|c|c|c|c|}
\hline & & \multicolumn{8}{|c|}{ Número de tubos de corriente } \\
\hline & & 9 & 10 & 11 & 12 & 13 & 14 & 15 & 16 \\
\hline \multirow{20}{*}{ 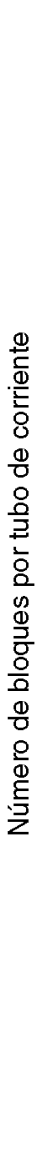 } & 1 & 0.0000003 & 0.0000003 & 0.0000003 & 0.0000003 & 0.0000003 & 0.0000003 & 0.0000003 & 0.0000003 \\
\hline & 2 & 0.0000011 & 0.0000011 & 0.0000011 & 0.0000011 & 0.0000011 & 0.0000011 & 0.0000011 & 0.0000011 \\
\hline & 3 & 0.0000042 & 0.0000042 & 0.0000042 & 0.0000042 & 0.0000042 & 0.0000042 & 0.0000042 & 0.0000042 \\
\hline & 4 & 0.0000151 & 0.0000151 & 0.0000151 & 0.0000151 & 0.0000151 & 0.0000151 & 0.0000150 & 0.0000150 \\
\hline & 5 & 0.0000532 & 0.0000532 & 0.0000532 & 0.0000532 & 0.0000532 & 0.0000532 & 0.0000532 & 0.0000532 \\
\hline & 6 & 0.0001870 & 0.0001870 & 0.0001870 & 0.0001870 & 0.0001870 & 0.0001870 & 0.0001870 & 0.0001870 \\
\hline & 7 & 0.0006573 & 0.0006573 & 0.0006572 & 0.0006572 & 0.0006572 & 0.0006572 & 0.0006571 & 0.0006571 \\
\hline & 8 & 0.0023085 & 0.0023085 & 0.0023063 & 0.0023063 & 0.0023047 & 0.0023047 & 0.0023038 & 0.0023038 \\
\hline & 9 & 0.0080568 & 0.0080568 & 0.0079705 & 0.0079705 & 0.0079080 & 0.0079080 & 0.0078753 & 0.0078753 \\
\hline & 10 & 0.0243041 & 0.0243041 & 0.0229862 & 0.0229862 & 0.0221844 & 0.0221844 & 0.0218032 & 0.0218032 \\
\hline & 11 & 0.0405515 & 0.0405515 & 0.0380019 & 0.0380019 & 0.0364609 & 0.0364609 & 0.0357310 & 0.0357310 \\
\hline & 12 & 0.0462997 & 0.0462997 & 0.0436660 & 0.0436660 & 0.0420642 & 0.0420642 & 0.0413025 & 0.0413025 \\
\hline & 13 & 0.0479509 & 0.0479510 & 0.0453151 & 0.0453151 & 0.0437117 & 0.0437117 & 0.0429492 & 0.0429492 \\
\hline & 14 & 0.0484212 & 0.0484212 & 0.0457854 & 0.0457855 & 0.0441819 & 0.0441819 & 0.0434193 & 0.0434193 \\
\hline & 15 & 0.0485551 & 0.0485551 & 0.0459192 & 0.0459192 & 0.0443157 & 0.0443157 & 0.0435532 & 0.0435532 \\
\hline & 16 & 0.0485932 & 0.0485932 & 0.0459573 & 0.0459573 & 0.0443538 & 0.0443538 & 0.0435913 & 0.0435913 \\
\hline & 17 & 0.0486040 & 0.0486040 & 0.0459681 & 0.0459681 & 0.0443647 & 0.0443647 & 0.0436021 & 0.0436021 \\
\hline & 18 & 0.0486071 & 0.0486071 & 0.0459712 & 0.0459712 & 0.0443677 & 0.0443677 & 0.0436052 & 0.0436052 \\
\hline & 19 & 0.0486080 & 0.0486080 & 0.0459721 & 0.0459721 & 0.0443686 & 0.0443686 & 0.0436061 & 0.0436061 \\
\hline & 20 & 0.0486082 & 0.0486082 & 0.0459723 & 0.0459723 & 0.0443689 & 0.0443689 & 0.0436063 & 0.0436063 \\
\hline
\end{tabular}

Tabla 5. Fracción areal de cada tubo de corriente para 16 tubos de corriente (parte 1)

\begin{tabular}{cccccccc}
\hline \multicolumn{7}{c}{ Número de tubos de corriente } \\
\hline $\mathbf{1}$ & $\mathbf{2}$ & $\mathbf{3}$ & $\mathbf{4}$ & $\mathbf{5}$ & $\mathbf{6}$ & $\mathbf{7}$ & $\mathbf{8}$ \\
\hline 0.1343376 & 0.1343376 & 0.0712345 & 0.0712345 & 0.0591854 & 0.0591854 & 0.0526867 & 0.0526867 \\
\hline
\end{tabular}

Tabla 6. Fracción areal de cada tubo de corriente para 16 tubos de corriente (parte 2)

\begin{tabular}{cccccccc}
\hline \multicolumn{10}{c}{ Número de tubos de corriente } \\
\hline $\mathbf{9}$ & 10 & $\mathbf{1 1}$ & $\mathbf{1 2}$ & $\mathbf{1 3}$ & $\mathbf{1 4}$ & $\mathbf{1 5}$ & $\mathbf{1 6}$ \\
\hline 0.0486082 & 0.0486082 & 0.0459723 & 0.0459723 & 0.0443689 & 0.0443689 & 0.0436063 & 0.0436063 \\
\hline
\end{tabular}

Por último, en las tablas 7, 8 y 9 se presentan los valores de la fracción areal de cada bloque en cada tubo de corriente, y en las tablas 10, 11 y 12, la fracción areal de cada tubo de corriente para veinticuatro tubos de corriente. 
Tabla 7. Fracción areal de cada bloque en cada tubo de corriente para 24 tubos de corriente (parte 1)

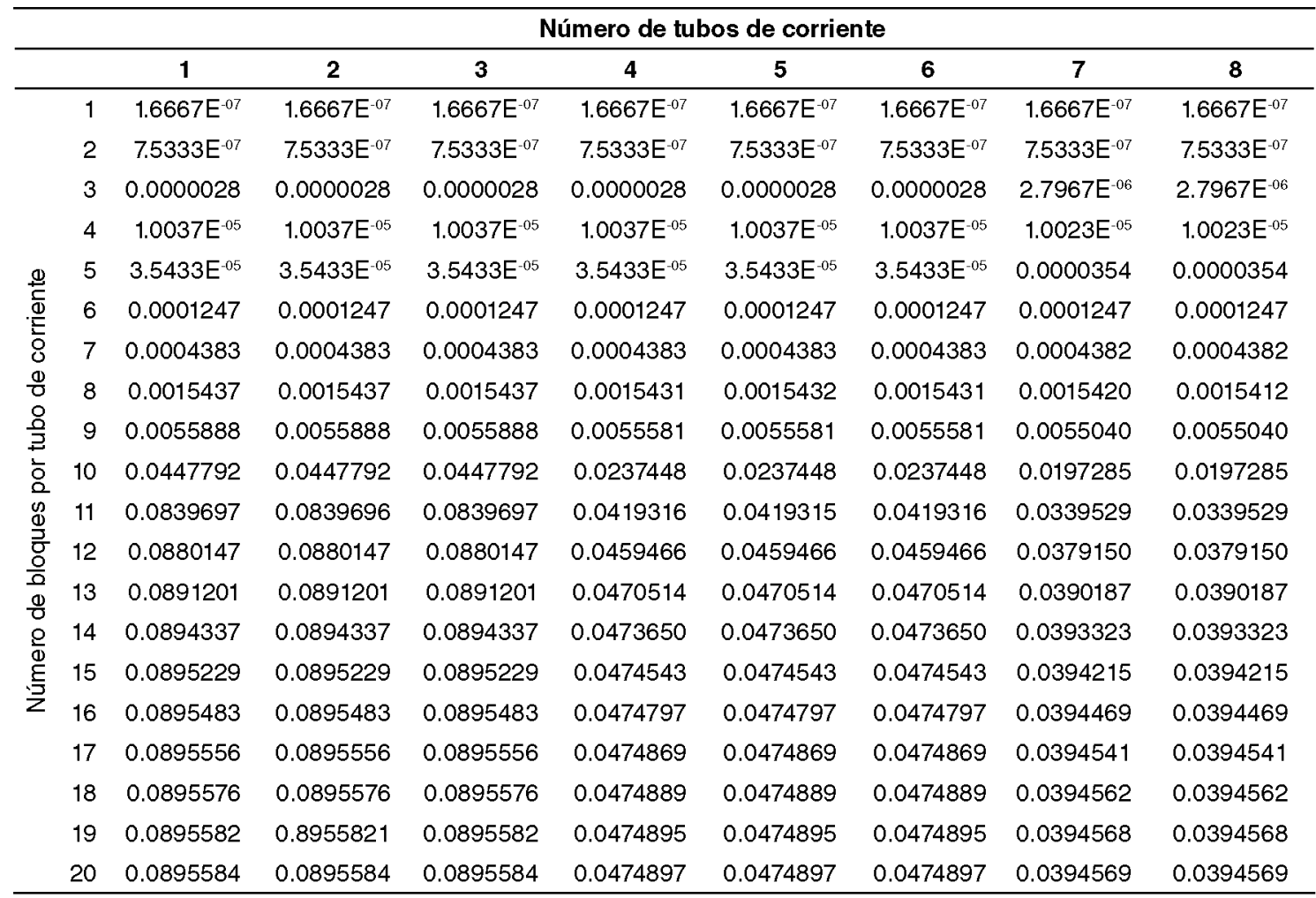

Tabla 8. Fracción areal de cada bloque en cada tubo de corriente para 24 tubos de corriente (parte 2)

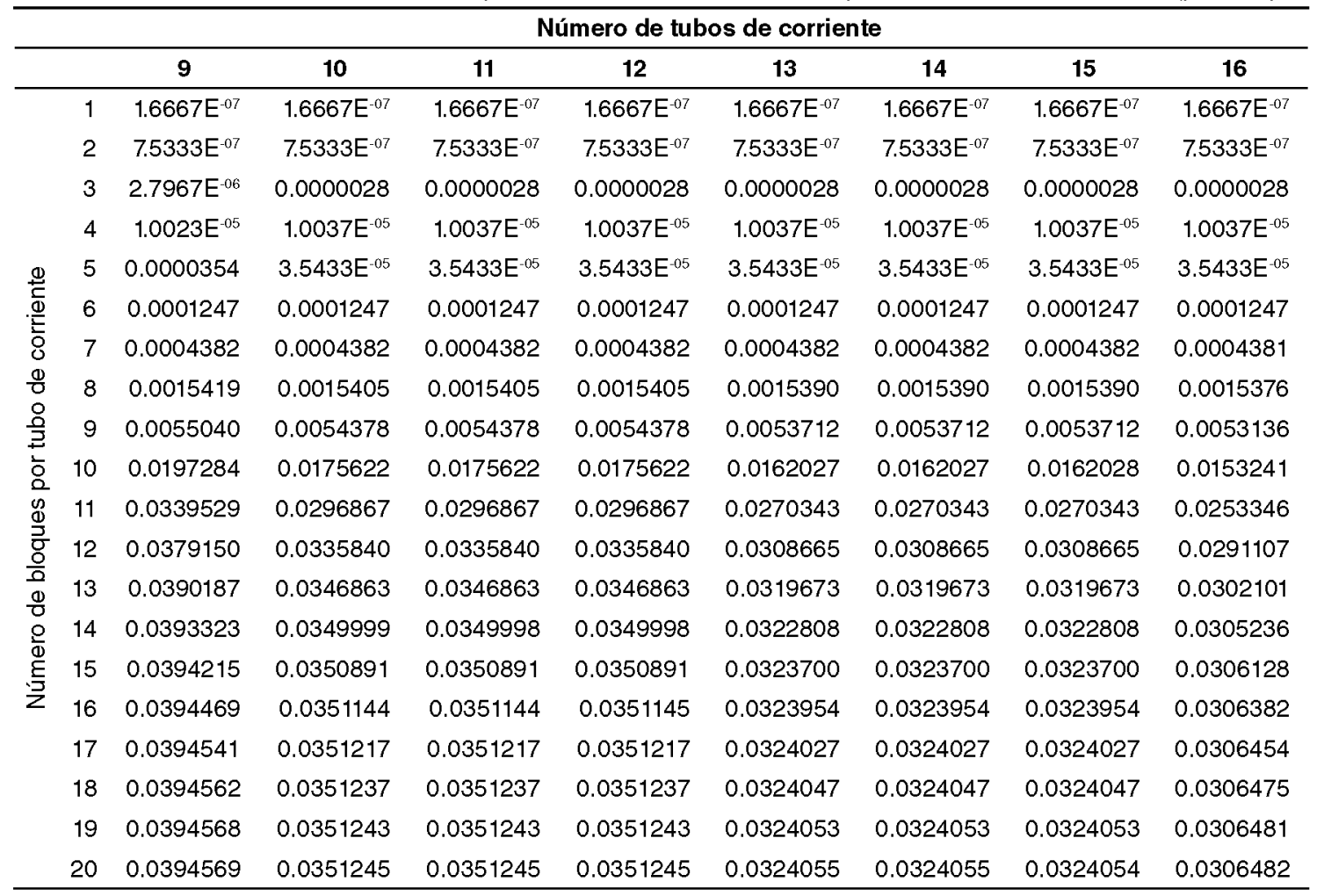


Tabla 9. Fracción areal de cada bloque en cada tubo de corriente para 24 tubos de corriente (parte 3)

\begin{tabular}{|c|c|c|c|c|c|c|c|c|c|}
\hline & & \multicolumn{8}{|c|}{ Número de tubos de corriente } \\
\hline & & 17 & 18 & 19 & 20 & 21 & 22 & 23 & 24 \\
\hline \multirow{20}{*}{ 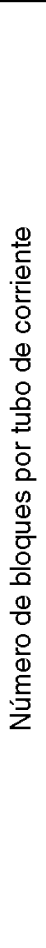 } & 1 & $1.6667 \mathrm{E}^{-07}$ & $1.6667 \mathrm{E}^{-07}$ & $1.6667 \mathrm{E}^{-07}$ & $1.6667 \mathrm{E}^{-07}$ & $1.6667 \mathrm{E}^{-07}$ & $1.6667 \mathrm{E}^{-07}$ & $1.6667 \mathrm{E}^{-07}$ & $1.6667 \mathrm{E}^{-07}$ \\
\hline & 2 & $7.5333 \mathrm{E}^{-07}$ & $7.5333 \mathrm{E}^{-07}$ & $7.5333 \mathrm{E}^{-07}$ & $7.5333 \mathrm{E}^{-07}$ & $7.5333 \mathrm{E}^{-07}$ & $7.5333 \mathrm{E}^{-07}$ & $7.5333 \mathrm{E}^{-07}$ & $7.5333 \mathrm{E}^{-07}$ \\
\hline & 3 & 0.0000028 & 0.0000028 & 0.0000028 & 0.0000028 & 0.0000028 & 0.0000028 & 0.0000028 & 0.0000028 \\
\hline & 4 & $1.0037 \mathrm{E}^{-05}$ & $1.0037 \mathrm{E}^{-05}$ & $1.0037 \mathrm{E}^{-05}$ & $1.0037 \mathrm{E}^{-05}$ & $1.0037 \mathrm{E}^{-05}$ & $1.0037 \mathrm{E}^{-05}$ & $1.0037 \mathrm{E}^{-05}$ & $1.0037 \mathrm{E}^{-05}$ \\
\hline & 5 & $3.5433 \mathrm{E}^{-05}$ & $3.5433 E^{-05}$ & $3.5433 E^{-05}$ & $3.5433 E^{-05}$ & $3.5433 E^{-05}$ & $3.5433 \mathrm{E}^{-05}$ & $3.5433 E^{-05}$ & $3.5433 E^{-05}$ \\
\hline & 6 & 0.0001247 & 0.0001247 & 0.0001247 & 0.0001247 & 0.0001247 & 0.0001247 & 0.0001247 & 0.0001247 \\
\hline & 7 & 0.0004381 & 0.0004381 & 0.0004381 & 0.0004381 & 0.0004381 & 0.0004381 & 0.0004381 & 0.0004381 \\
\hline & 8 & 0.0015376 & 0.0015376 & 0.0015365 & 0.0015365 & 0.0015365 & 0.0015358 & 0.0015359 & 0.0015359 \\
\hline & 9 & 0.0053136 & 0.0053136 & 0.0052712 & 0.0052720 & 0.0052720 & 0.0052502 & 0.0052502 & 0.0052502 \\
\hline & 10 & 0.0153241 & 0.0153241 & 0.0147896 & 0.0147896 & 0.0147896 & 0.0145354 & 0.0145354 & 0.0145354 \\
\hline & 11 & 0.0253346 & 0.0253346 & 0.0243073 & 0.0243073 & 0.0243073 & 0.0238207 & 0.0238207 & 0.0238207 \\
\hline & 12 & 0.0291107 & 0.0291107 & 0.0280428 & 0.0280428 & 0.0280428 & 0.0275350 & 0.0275350 & 0.0275350 \\
\hline & 13 & 0.0302101 & 0.0302101 & 0.0291411 & 0.0291411 & 0.0291411 & 0.0286328 & 0.0286328 & 0.0286328 \\
\hline & 14 & 0.0305236 & 0.0305236 & 0.0294546 & 0.0294546 & 0.0294546 & 0.0289462 & 0.0289462 & 0.0289462 \\
\hline & 15 & 0.0306128 & 0.0306128 & 0.0295438 & 0.0295438 & 0.0295438 & 0.0290354 & 0.0290354 & 0.2903544 \\
\hline & 16 & 0.0306382 & 0.0306382 & 0.0295692 & 0.0295692 & 0.0295692 & 0.0290608 & 0.0290608 & 0.0290608 \\
\hline & 17 & 0.0306454 & 0.0306454 & 0.0295764 & 0.0295764 & 0.0295764 & 0.0290681 & 0.0290681 & 0.0290681 \\
\hline & 18 & 0.0306475 & 0.0306475 & 0.0295785 & 0.0295785 & 0.0295785 & 0.0290701 & 0.0290701 & 0.0290701 \\
\hline & 19 & 0.0306481 & 0.0306481 & 0.0295791 & 0.0295791 & 0.0295791 & 0.0290707 & 0.0290707 & 0.0290707 \\
\hline & 20 & 0.0306482 & 0.0306482 & 0.0295792 & 0.0295792 & 0.0295792 & 0.0290709 & 0.0290709 & 0.0290709 \\
\hline
\end{tabular}

Tabla 10. Fracción areal de cada tubo de corriente para 24 tubos de corriente (parte 1)

\begin{tabular}{cccccccc}
\hline \multicolumn{7}{c}{ Número de tubos de corriente } \\
\hline $\mathbf{1}$ & $\mathbf{2}$ & $\mathbf{3}$ & $\mathbf{4}$ & $\mathbf{5}$ & $\mathbf{6}$ & $\mathbf{7}$ & $\mathbf{8}$ \\
\hline 0.0895584 & 0.0895584 & 0.0895584 & 0.0474897 & 0.0474897 & 0.0474897 & 0.0394569 & 0.0394569 \\
\hline
\end{tabular}

Tabla 11. Fracción areal de cada tubo de corriente para 24 tubos de corriente (parte 2)

\begin{tabular}{cccccccc}
\hline \multicolumn{7}{c}{ Número de tubos de corriente } \\
\hline $\mathbf{9}$ & $\mathbf{1 0}$ & $\mathbf{1 1}$ & $\mathbf{1 2}$ & $\mathbf{1 3}$ & $\mathbf{1 4}$ & $\mathbf{1 5}$ & $\mathbf{1 6}$ \\
\hline 0.0394569 & 0.0351245 & 0.0351245 & 0.0351245 & 0.0324054 & 0.0324055 & 0.0324055 & 0.0306482 \\
\hline
\end{tabular}

Tabla 12. Fracción areal de cada tubo de corriente para 24 tubos de corriente (parte 3 )

\begin{tabular}{cccccccc}
\hline \multicolumn{7}{c}{ Número de tubos de corriente } \\
\hline $\mathbf{1 7}$ & $\mathbf{1 8}$ & $\mathbf{1 9}$ & $\mathbf{2 0}$ & $\mathbf{2 1}$ & $\mathbf{2 2}$ & $\mathbf{2 3}$ & $\mathbf{2 4}$ \\
\hline 0.0306482 & 0.0306482 & 0.0295792 & 0.0295792 & 0.0295792 & 0.0290709 & 0.0290709 & $0, .2907088$ \\
\hline
\end{tabular}

\section{Resultados}

La estructura general de la ventana principal de la aplicación CHP se puede observar en la figura 1. CHP es una herramienta que realiza la predicción para un esquema de desarrollo, mediante un procedimiento que comprende tres etapas: 
a. Información minima requerida. El modelo predictivo no necesita datos detallados del campo de estudio (como sí los requiere un modelo de simulación numérica); sin embargo, de acuerdo con la estructura de la aplicación, es necesario tener la información solicitada en las siguientes secciones: generalidades del modelo, información de entrada, estratificación y datos del proceso EOR.

b. Resultados. Luego de realizar la evaluación del proceso seleccionado, se pueden observar los resultados en tablas. Además, el comportamiento con respecto al tiempo de las variables principales estudiadas (tasa de producción, producción acumulada y factor de recobro) se presenta en gráficas.

c. Ajuste. La herramienta ofrece la posibilidad de comparar los resultados del modelo realizado con los resultados generados por otro método de evaluación (se debe importar la historia a la aplicación).

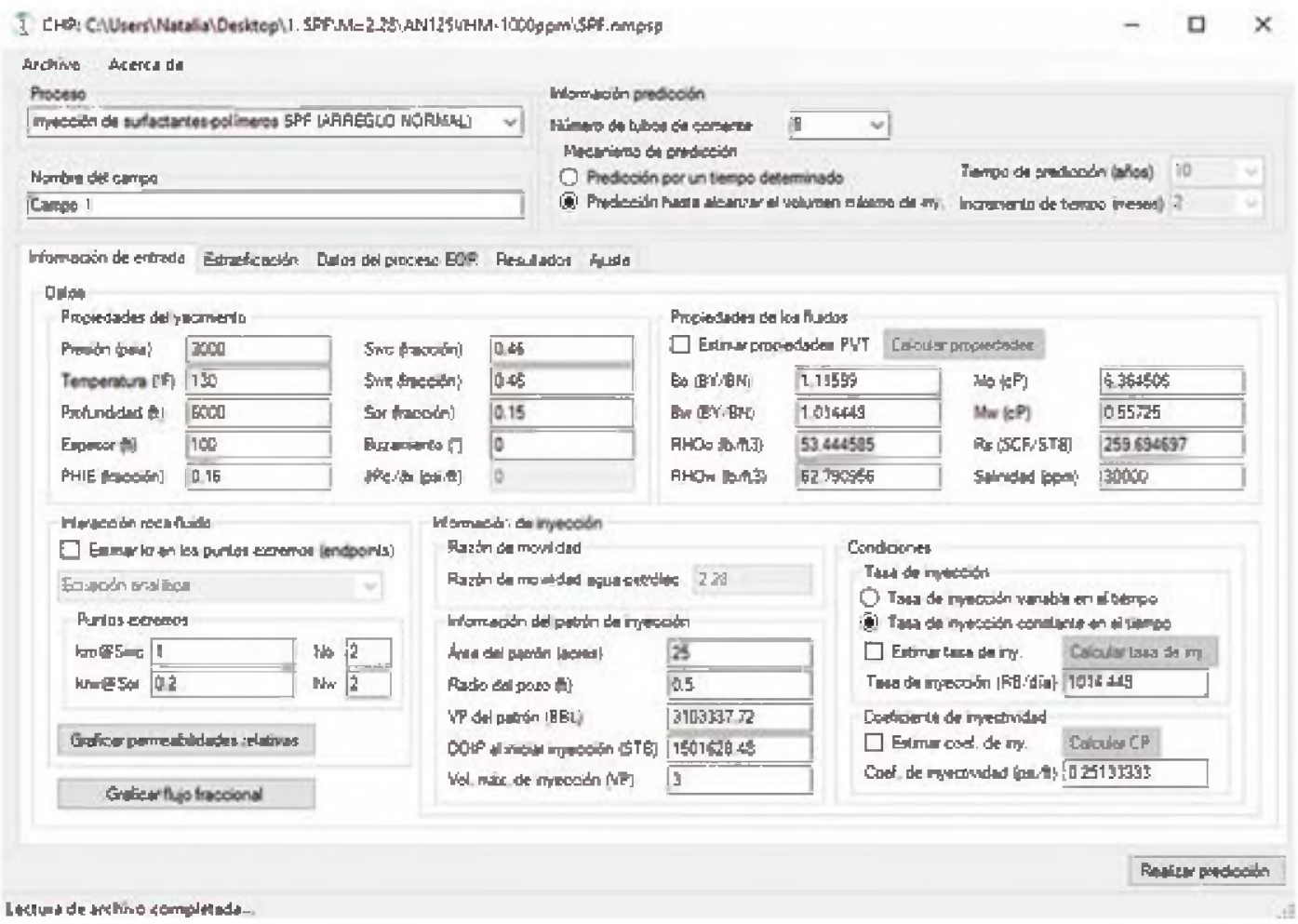

Figura 1. Interfaz de la ventana principal. Aplicación CHP desarrollada por los autores.

\section{Generalidades del modelo}

La parte superior de la interfaz tiene información general para llevar a cabo la predicción. Para la predicción es necesario definir ciertos parámetros.

\section{Proceso}

Los modelos predictivos codificados en la herramienta, que se pueden utilizar en la evaluación de un esquema de desarrollo, son: inyección de agua WF (DOE y MEMV, 1986b), inyección de polímeros PF (DOE y MEMV, 1986b), inyección de surfactantes-polímeros SPF (DOE y MEMV, 
1986a), inyección de polímeros PF (arreglo normal), inyección de polímeros PF (arreglo invertido), inyección de surfactantes SF (arreglo normal) e inyección de surfactantes-polímeros SPF (arreglo normal).

\section{Nombre del campo}

Para realizar la predicción y guardar, se debe ingresar el nombre del campo que será objeto de estudio.

\section{Número de tubos de corriente}

La predicción se basa en una aproximación que utiliza tubos de corriente. Cada capa del reservorio se divide en el número de tubos de corriente seleccionado en la aplicación $(1,4,8,16 \circ 24)$.

\section{Mecanismo de predicción}

El caso planteado se puede evaluar hasta alcanzar el volumen máximo de inyección o por un período de tiempo determinado; para la segunda opción se debe especificar tiempo e incremento de tiempo.

\section{INFORMACIÓN DE ENTRADA}

En esta ventana se deben ingresar los datos generales del modelo. Aunque se realiza una predicción con poca información, se presentan cinco subdivisiones principales y cada una de ellas requiere cierto número de datos.

\section{Propiedades del yacimiento}

Se deben especificar las características de la formación y propiedades de la roca: presión, temperatura, profundidad, espesor, porosidad efectiva, saturación de agua connata, saturación promedio de agua al iniciar la inyección, saturación de petróleo residual, buzamiento y gradiente de presión capilar.

\section{Propiedades de los fluidos}

En esta sección se deben definir las características de los fluidos que se producen en el yacimiento (agua y petróleo): factor volumétrico del petróleo, factor volumétrico del agua, densidad del petróleo, densidad del agua, viscosidad del petróleo, viscosidad del agua, relación gas disuelto-petróleo y salinidad. Las propiedades del gas no se tienen en cuenta porque no afectan significativamente el pronóstico.

La aplicación CHP permite que las propiedades sean calculadas mediante correlaciones numéricas. En la ventana que se puede observar en la figura 2, se deben ingresar datos que son indispensables para realizar la estimación: gravedad API, gravedad específica del gas, relación gas disuelto-petróleo (es posible estimar esta variable a partir de la presión de burbuja cuando no se tiene la información) y condiciones del separador en superficie (presión y temperatura). En la ventana de la figura 3 se seleccionan las correlaciones para el agua y para el petróleo. 


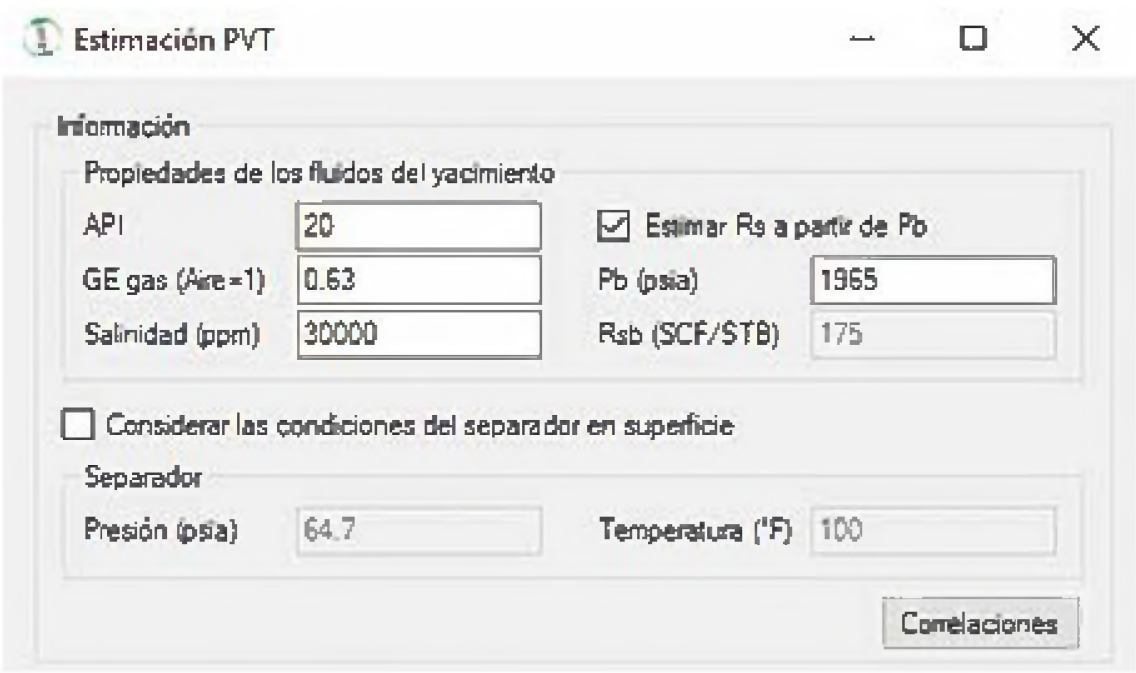

Figura 2. Ventana de propiedades requeridas para estimar las propiedades de los fluidos. Aplicación CHP desarrollada por los autores.

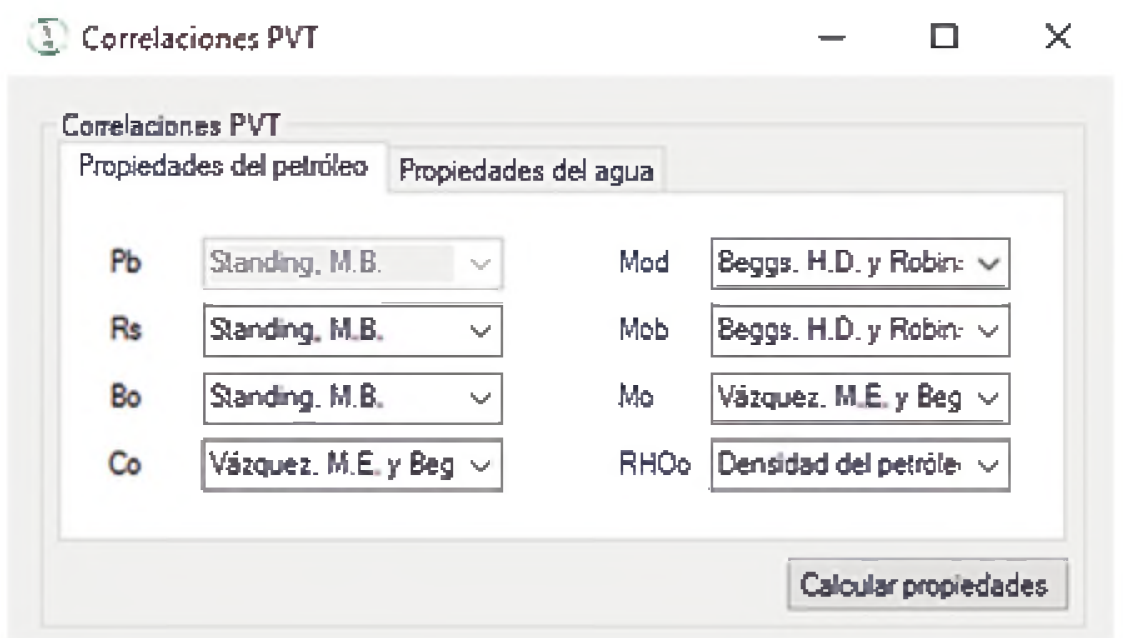

Figura 3. Ventana de correlaciones para calcular las propiedades de los fluidos, petróleo y agua. Aplicación CHP desarrollada por los autores.

\section{Interacción roca-fluido}

En esta sección es indispensable conocer los puntos extremos de las curvas de permeabilidades relativas: permeabilidad al agua a la saturación de petróleo residual (krw@Sor) y permeabilidad al petróleo a la saturación de agua connata (kro@Swc).

Cuando hay información disponible de los puntos extremos, se deben ingresar los exponentes de las curvas de permeabilidades relativas al petróleo y al agua $\left(\mathrm{n}_{\mathrm{o}}\right.$ y $\mathrm{n}_{\mathrm{w}}$, respectivamente); en este caso, se obtienen resultados derivados de la ecuación analítica. Por otro lado, si no hay datos disponibles, se pueden determinar por medio de correlaciones que están en función de la saturación de agua connata y la saturación de petróleo residual. Asimismo, CHP reúne cinco correlaciones 
para sistemas mojados por agua y por petróleo, que fueron planteadas por: Corey (Honarpour, Koederitz y Harvey, 1986, p. 32), Smith (Paris, 2001, p. 54), Wyllie y Gardner (Ahmed, 2006, p. 297), Wyllie y Gardner (Ahmed, 2006, p. 297) y Pirson (Ahmed, 2006, p. 298), respectivamente.

La aplicación permite crear la gráfica de permeabilidades relativas (ver figura 4) y una tabla con los datos respectivos (ver figura 5) en una ventana adicional, desde donde se puede cambiar la correlación para determinar las propiedades de interacción roca-fluido (si la información no está disponible), considerando las premisas correspondientes.

En el caso de una predicción de inyección de polímeros, inyección de surfactantes o inyección de surfactantes-polímeros, también se cuenta con la posibilidad de comparar las curvas de permeabilidades relativas de los procesos químicos con las de la inyección de agua.

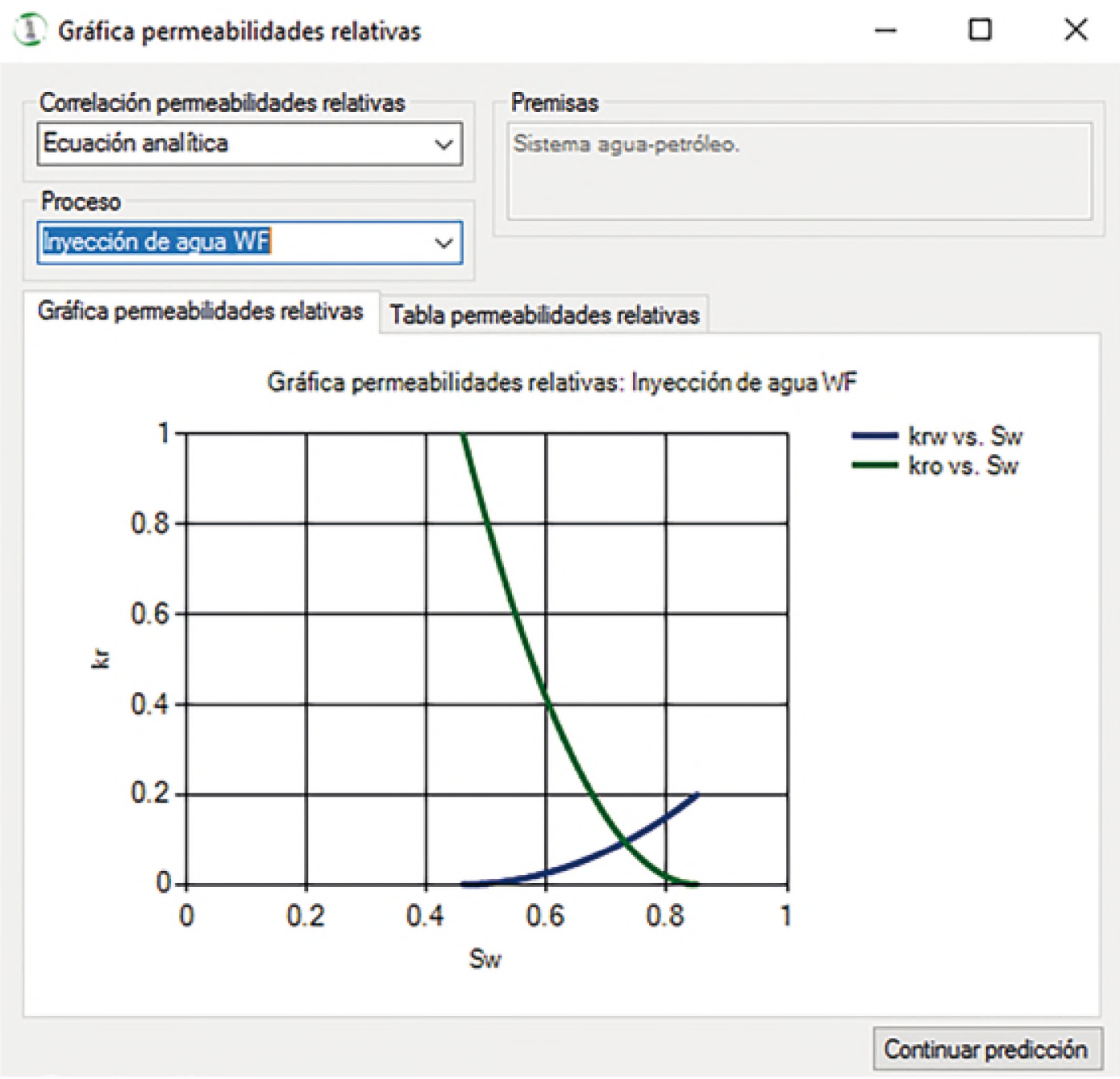

Figura 4. Ventana de curva de permeabilidades relativas al agua y al petróleo. Aplicación CHP desarrollada por los autores. 


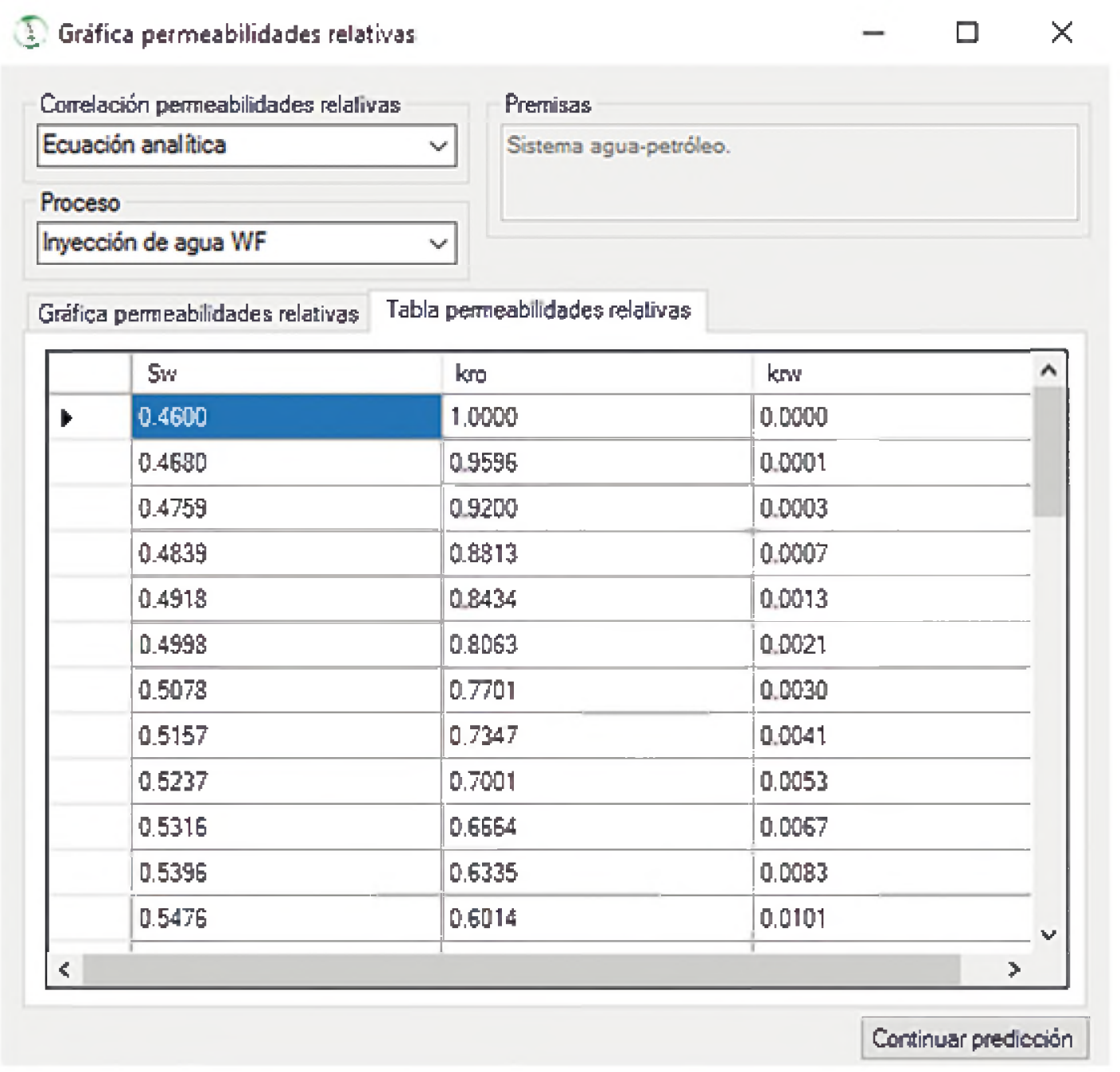

Figura 5. Ventana de tabla de valores de permeabilidades relativas al agua y al petróleo. Aplicación CHP desarrollada por los autores.

\section{Flujo fraccional}

De igual manera, se puede generar la curva de flujo fraccional a partir de los datos de entrada mínimos que requiere el código en la ventana principal. En esta sección, como en la de permeabilidades relativas, se puede cambiar la correlación (si no se conocen los puntos extremos de las curvas de permeabilidades relativas) para obtener los resultados y se puede comparar los procesos de inyección química con la inyección de agua. La gráfica de la curva fw vs. Sw se puede observar en la figura 6, y la tabla con los datos correspondientes, en la figura 7 .

Igualmente, se calculan las propiedades de la curva de flujo fraccional, que son resumidas en el cuadro de información obtenida: saturación de agua del frente, flujo fraccional del frente y saturación de agua promedio a la ruptura. 
I. Gráfica flujo fraccional

Conelación permeablidades relativas Ecuacion and hica

Proceso Injocción de polimeros PF

Grica flujo fraccional Tabla fujo tracoions:

Gráfica flujo fraccional inyección de polimeros PF

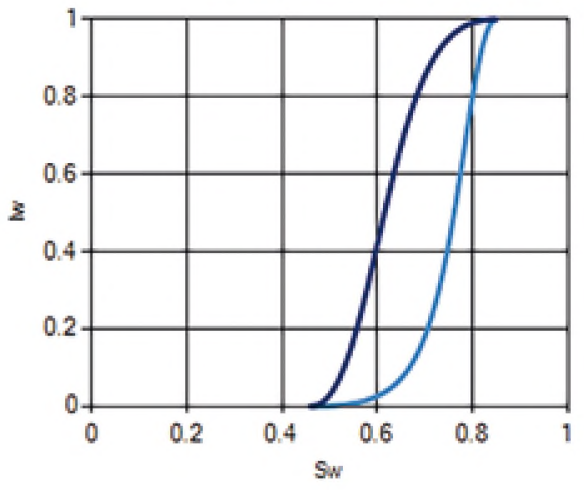

Irfomaclón obtenida

Sat 0.8341

fut 0.9793

$\checkmark$ Comparar cumas de fw ve. Sw $-\square \times$

\section{- fwvs, Sw(PF)}

Figura 6. Ventana de curva de flujo fraccional. Aplicación CHP desarrollada por los autores.

I Graflica ligho Iractions?

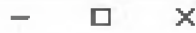

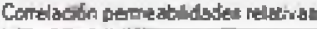
Ecuscibs anallica

Protede

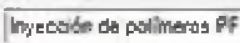

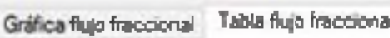

\begin{tabular}{|l|l|l|}
\hline & Sw & fw \\
\hline 0.4600 & 0.0000 \\
\hline 0.4680 & 0.0000 \\
\hline 0.4759 & 0.0002 \\
\hline 0.4839 & 0.0004 \\
\hline 0.4918 & 0.0007 \\
\hline 0.4998 & 0.0011 \\
\hline 0.5078 & 0.0017 \\
\hline 0.5157 & 0.0024 \\
\hline 0.5237 & 0.0082 \\
\hline 0.5316 & 0.0043 \\
\hline 0.5355 & 0.0056 \\
\hline 0.5476 & 0.0071 \\
\hline 0.5555 & 0.0089 \\
\hline
\end{tabular}

Conthturpredoción

Figura 7. Ventana de tabla de valores de flujo fraccional. Aplicación CHP desarrollada por los autores. 


\section{Información de inyección}

La última sección de los datos de entrada comprende la información propia del proceso de inyección. Se divide en tres áreas principales: razón de movilidad agua-petróleo, información del patrón de inyección y condiciones.

La herramienta determina la razón de movilidad agua-petróleo considerando la viscosidad del agua, la viscosidad del petróleo y las permeabilidades relativas en los puntos extremos.

La información del patrón de inyección incluye: área del patrón, radio del pozo, volumen poroso del patrón, petróleo original en sitio al iniciar la inyección y volumen máximo de inyección.

Con respecto a las condiciones de la inyección, se necesita: tasa de inyección, que puede ser constante o variable en el tiempo, y coeficiente de inyectividad, que se puede determinar por medio de una correlación y que depende de la profundidad del reservorio.

\section{ESTRATIFICACIÓN}

En la ventana se determinan las propiedades petrofísicas de cada una de las capas del reservorio (ver la figura 8), espesor, porosidad efectiva, saturación de agua inicial y permeabilidad, a partir de la siguiente información: número de capas, coeficiente de Dykstra-Parsons, permeabilidad horizontal y permeabilidad vertical.

Hay tres posibilidades para considerar el método de estratificación en el modelo predictivo diseñado: capas de igual espesor, capas de igual producto permeabilidad-espesor y modelo multicapas (cuando la información detallada por capas está disponible, esta opción permite ingresarla).

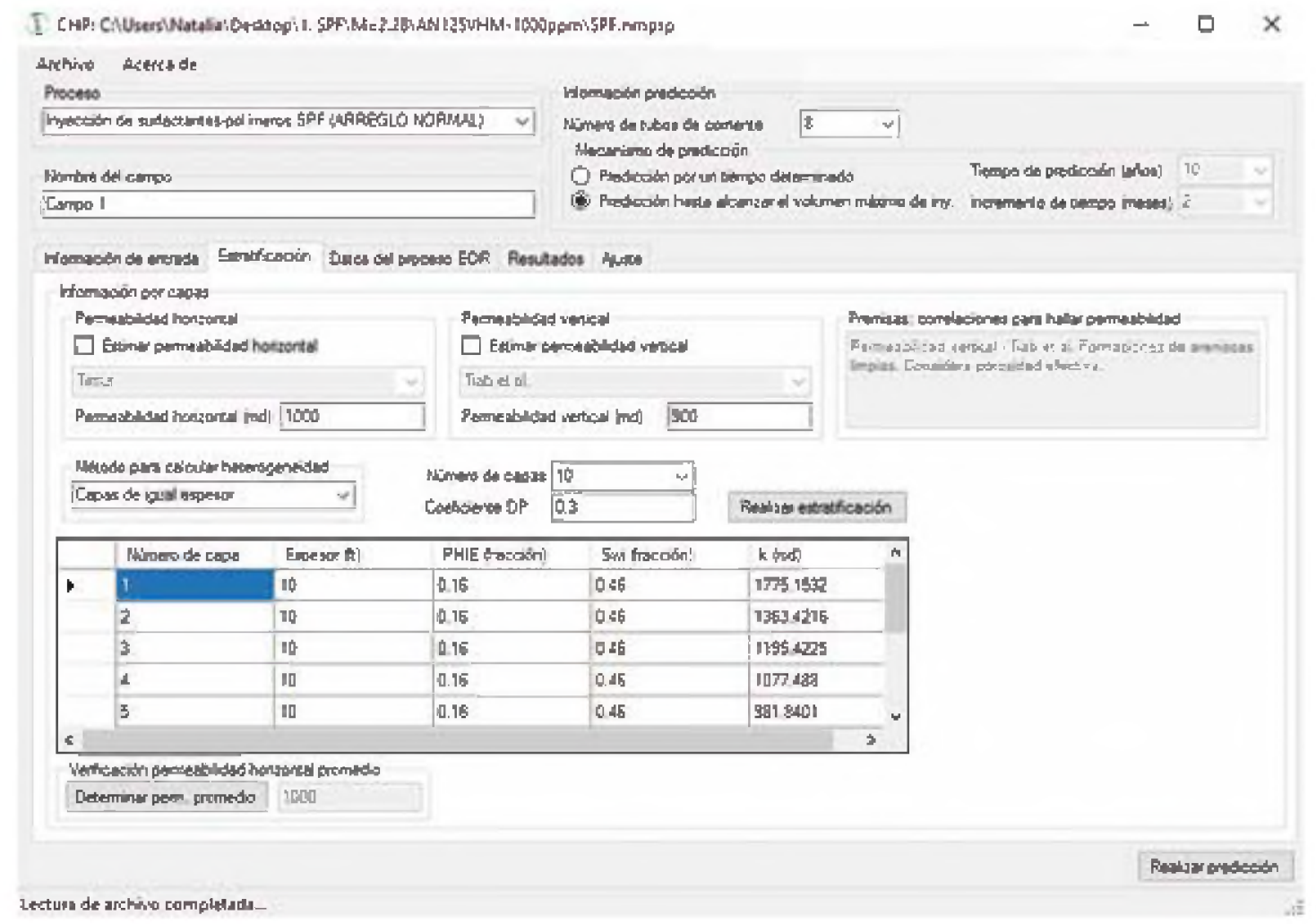

Figura 8. Ventana de propiedades petrofísicas de cada capa del reservorio. Aplicación CHP desarrollada por los autores. 


\section{DATOS DEL PROCESO EOR}

En esta ventana se especifican los datos de los procesos químicos que aplican en el caso de estudio. Como se ha dicho anteriormente, la aplicación permite analizar la inyección de polímeros, la inyección de surfactantes y la inyección de surfactantes-polímeros.

Para cada caso se necesitan diferentes datos de las propiedades, según los requerimientos del código para la evaluación. La figura 9 muestra la estructura general de esta ventana en la aplicación CHP.

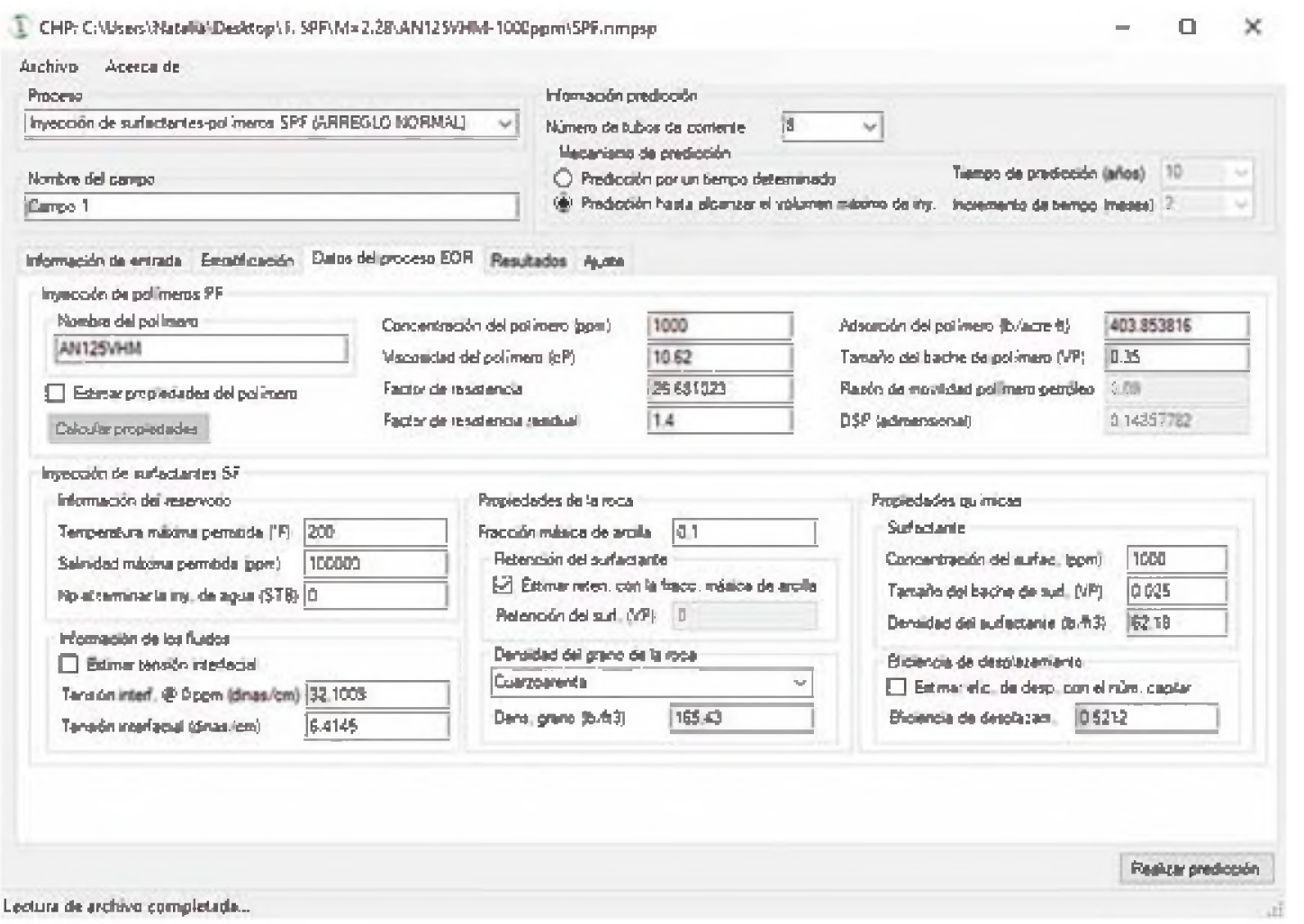

Figura 9. Ventana de propiedades de los químicos que se utilizan en el proceso de evaluación. Aplicación CHP desarrollada por los autores.

\section{Polímeros}

En este módulo se requiere: nombre, concentración, viscosidad, factor de resistencia, factor de resistencia residual, adsorción y tamaño del bache.

El programa calcula automáticamente la adsorción adimensional y la razón de movilidad polímero-petróleo.

\section{Surfactantes}

Esta información se organiza en cuatro módulos: información del reservorio, propiedades de los fluidos, propiedades de la roca y propiedades químicas.

- Información del reservorio: temperatura máxima, salinidad máxima y producción acumulada de petróleo al terminar la inyección de agua. 
- Propiedades de los fluidos: tensión interfacial a 0 ppm y tensión interfacial a la concentración del químico.

- Propiedades de la roca: fracción másica de arcilla, retención del surfactante (se puede calcular con una correlación en función de la fracción másica de arcilla) y densidad del grano de la roca.

- Propiedades quimicas: concentración, tamaño del bache, densidad y eficiencia de desplazamiento; esta última se puede calcular con el programa mediante una correlación que considera el número capilar, utilizando las curvas de desaturación capilar para areniscas Berea (DOE y MEMV, 1986a).

\section{Resultados}

Luego de realizar la predicción, los resultados son escritos en las tablas y se representan por medio de gráficas.

\section{Tablas}

\section{Detalles por capa}

Los datos que se obtienen son: saturación de agua inicial, flujo fraccional a la saturación de agua inicial, movilidad total relativa del banco de agua, saturación del banco de petróleo, flujo fraccional del banco de petróleo, velocidad adimensional del banco de petróleo, ruptura del banco de petróleo, movilidad total relativa del banco de petróleo/agua connata, saturación del frente de agua/polímero, flujo fraccional del frente de agua/polímero, velocidad adimensional del frente de agua/polímero, ruptura del frente de agua/polímero y movilidad total relativa del banco de agua/polímero (ver figura 10).

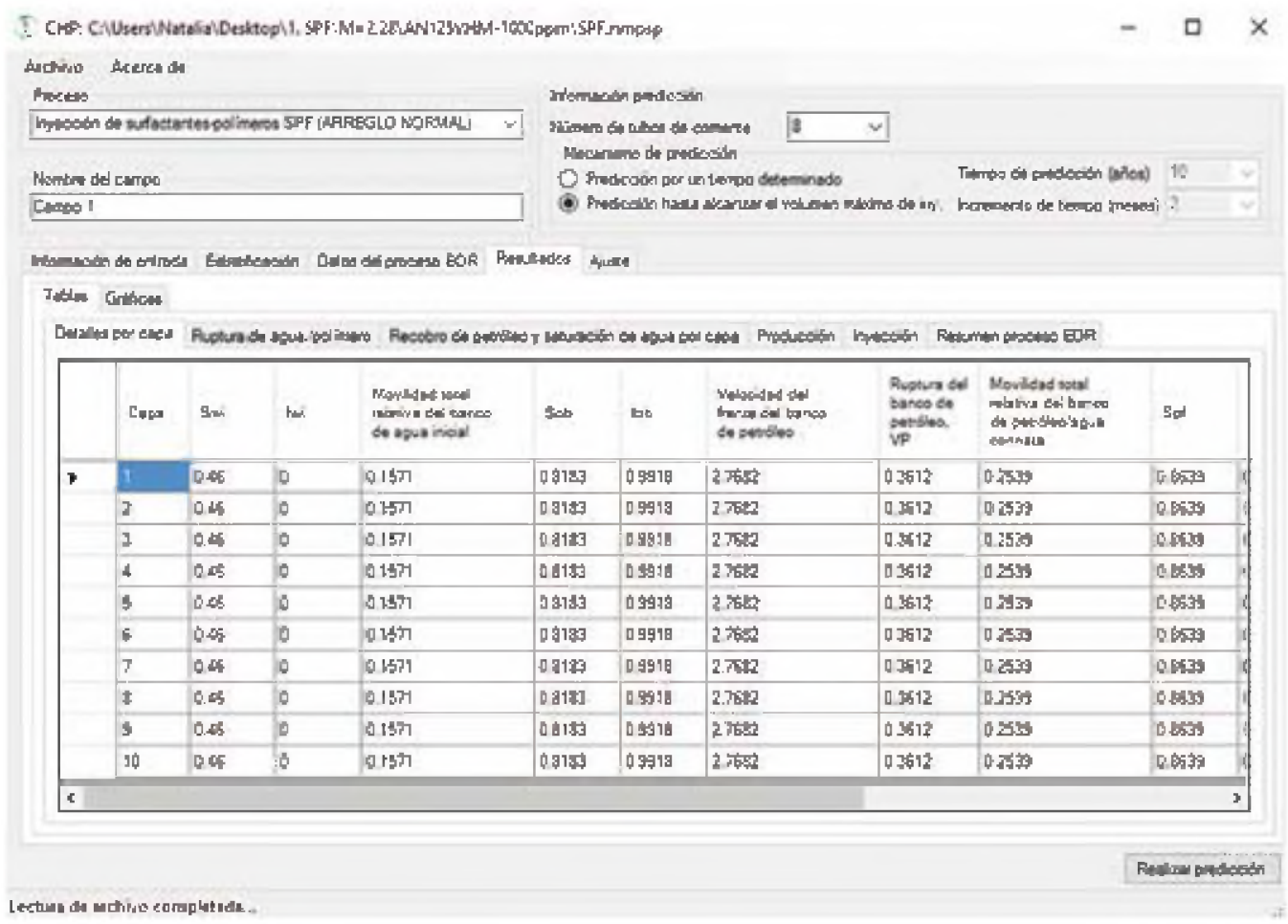

Figura 10. Ventana de resultados de los bancos en el proceso de inyección. Aplicación CHP desarrollada por los autores. 


\section{Ruptura de agua/polímero}

Se organizan los resultados de saturación después de presentarse la ruptura de la fase desplazante (ver figura 11): saturación de agua a XD = 1 (final del sistema), saturación promedio de agua desde $\mathrm{XD}=0$ hasta 1 , ruptura del frente de agua a $\mathrm{XD}=1$ en términos de volumen poroso y recobro de petróleo acumulado a la ruptura en términos de volumen poroso.

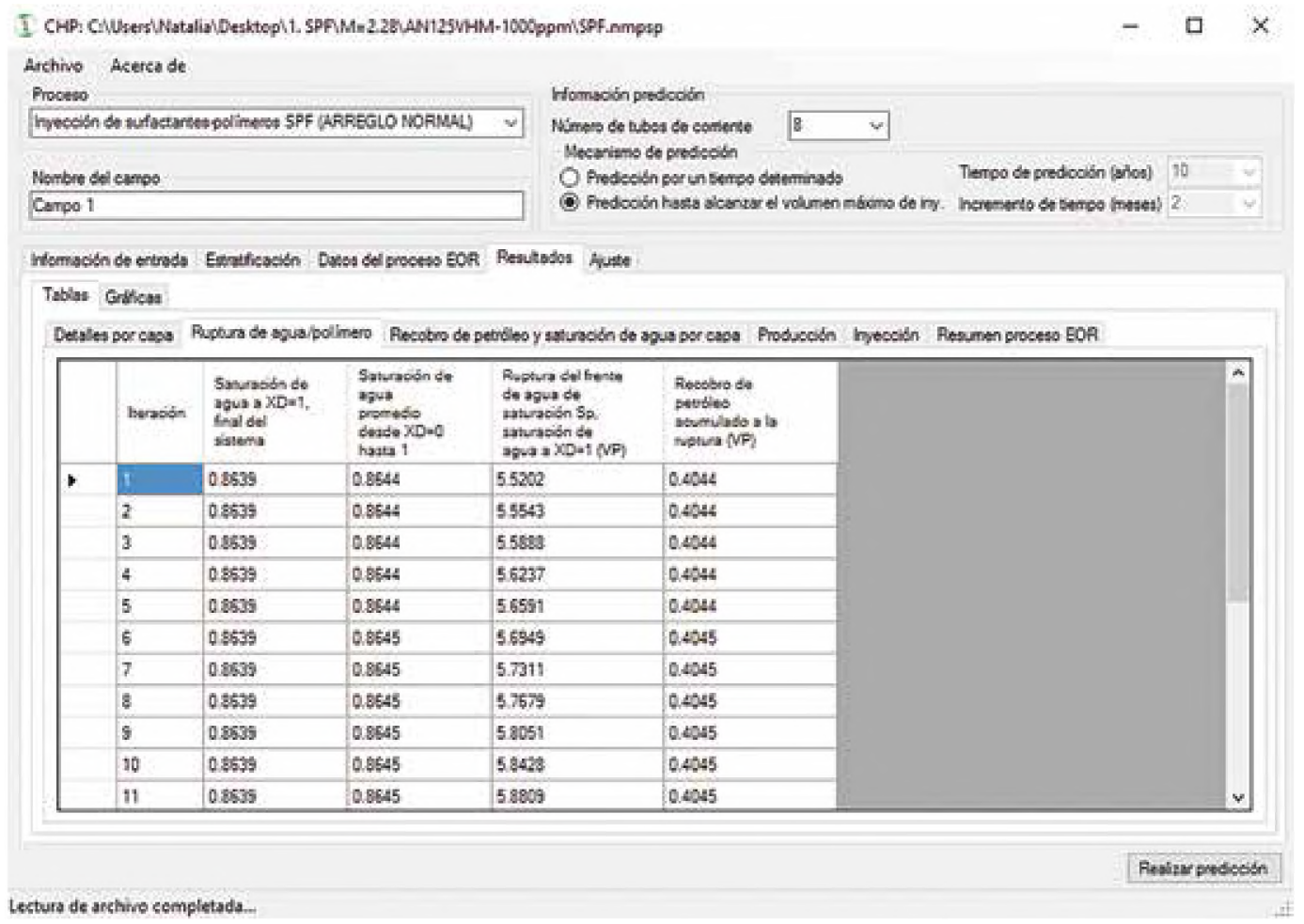

Figura 11. Ventana de resultados de propiedades a la ruptura de agua o del polímero. Aplicación CHP desarrollada por los autores.

\section{Recobro de petróleo y saturación de agua por capa}

Es posible seleccionar una capa específica para observar los resultados obtenidos con la predicción realizada, de acuerdo a la estructura que se muestra en la figura 12: paso de tiempo, tiempo real, tiempo adimensional (volumen inyectado), factor de recobro de petróleo en todos los tubos de corriente, índice de inyectividad, recobro de petróleo y saturación de agua.

\section{Producción}

La aplicación permite generar resultados de tiempo, tasa de petróleo, tasa de gas, tasa de agua, tasa líquida, petróleo acumulado, gas acumulado, agua acumulada, factor de recobro de petróleo y razón agua-petróleo (ver figura 13).

\section{Inyección}

Se obtienen resultados de tiempo, tiempo adimensional, tasa de agua, tasa de polímero, índice de inyectividad, agua acumulada y polímero acumulado (ver figura 14). 


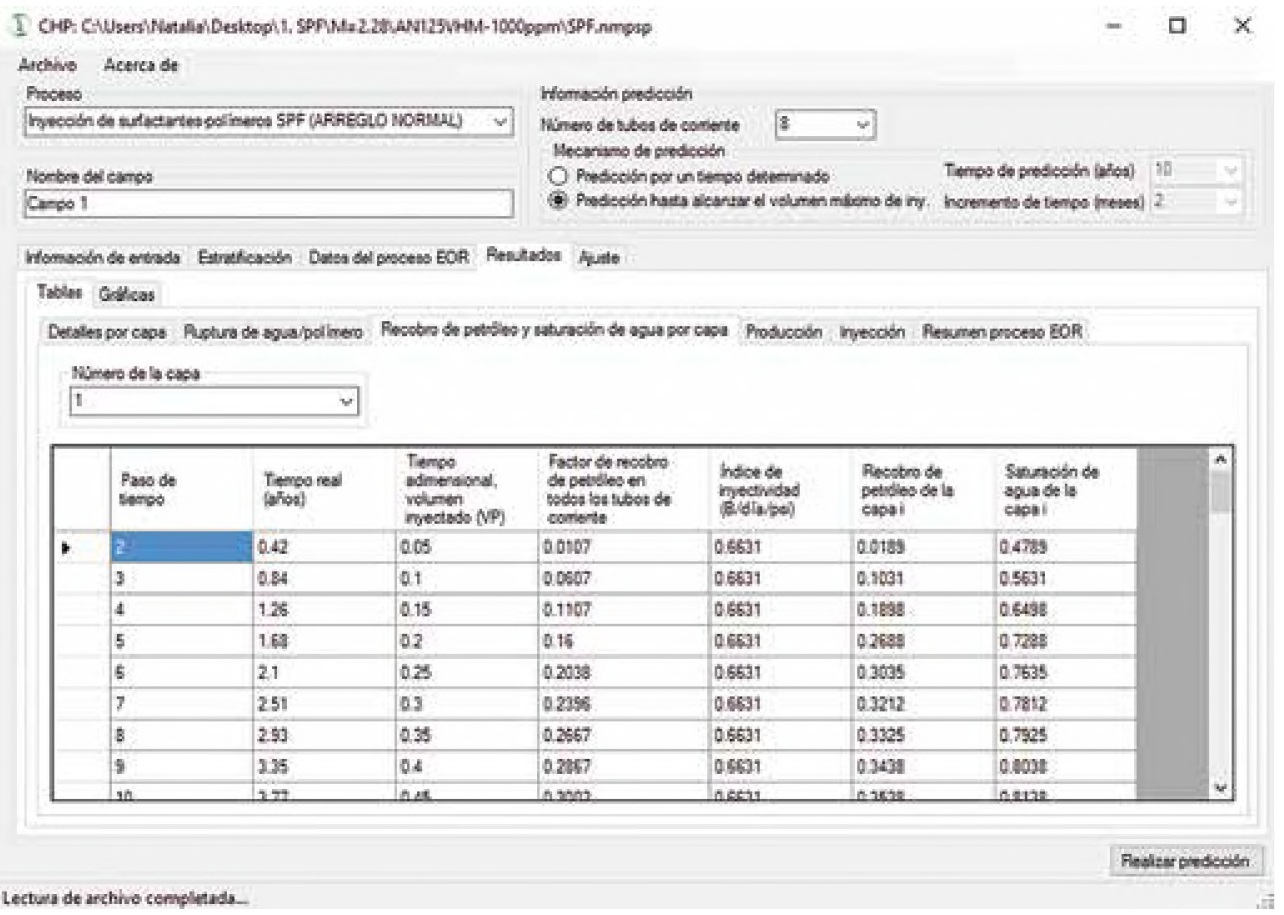

Figura 12. Ventana de resultados de una capa del reservorio seleccionada por cada paso de tiempo. Aplicación CHP desarrollada por los autores.

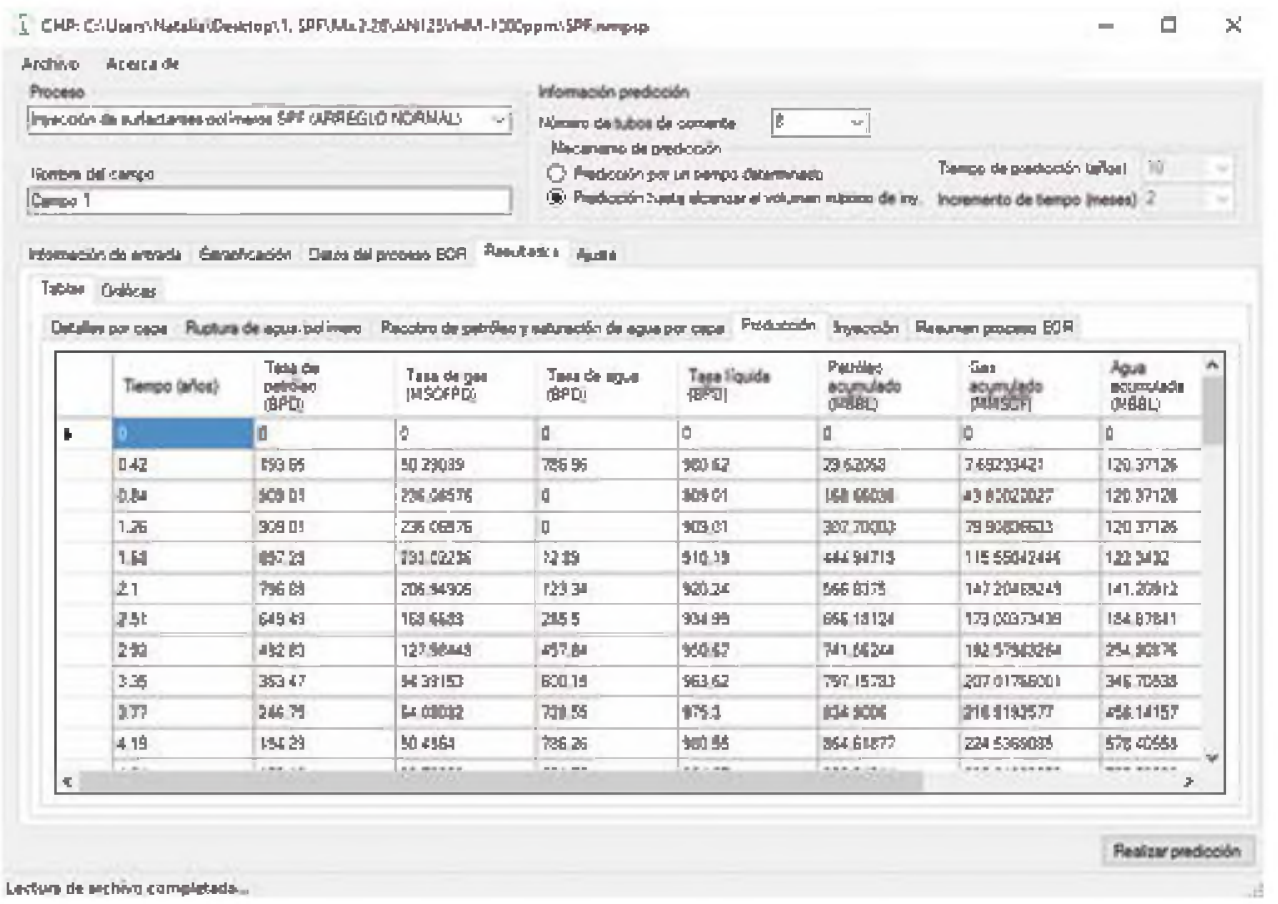

Figura 13. Ventana de resultados de producción por cada paso de tiempo. Aplicación CHP desarrollada por los autores. 


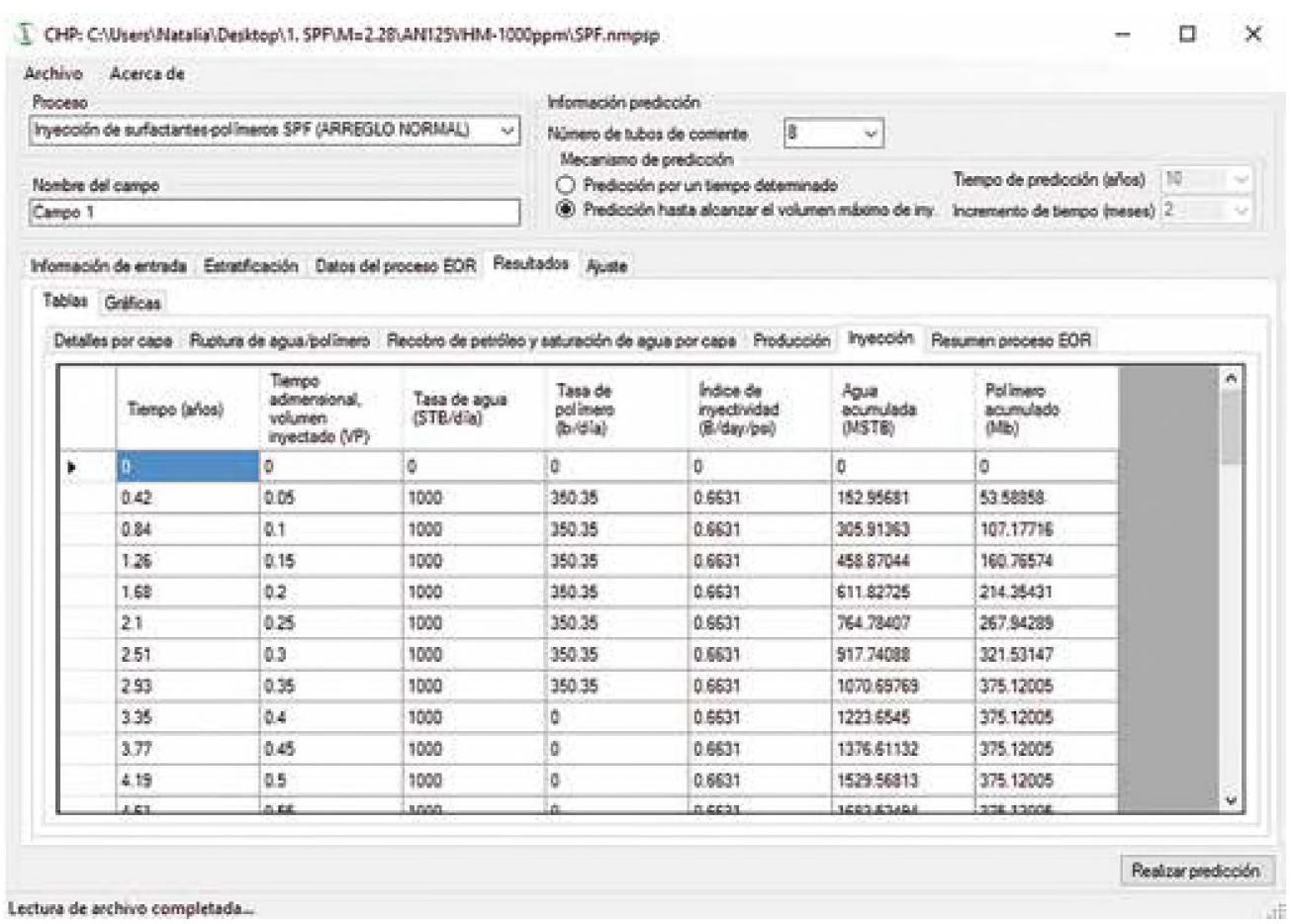

Figura 14. Ventana de resultados de inyección por cada paso de tiempo. Aplicación CHP desarrollada por los autores.

\section{Resumen proceso EOR}

En esta tabla se resumen los datos específicos de la predicción realizada en la aplicación para los procesos de inyección química (ver figura 15). Los datos que se obtiene son: número capilar del campo, eficiencia de desplazamiento, número de flujo cruzado, retención de surfactante, tamaño adimensional del tapón de surfactante, tamaño del tapón de surfactante, tamaño del bache de polímero, coeficiente de Dykstra-Parsons, razón de movilidad efectiva, capacidad de flujo de la capa m, capacidad de almacenamiento de la capa m, eficiencia de barrido vertical, eficiencia de barrido del bache de polímero, factor de comportamiento de flujo cruzado, eficiencia de recobro terciario de petróleo, volumen del tapón de surfactante en el patrón, concentración inicial de polímero, requerimiento de polímero en el patrón, velocidad adimensional del surfactante, velocidad adimensional del banco de petróleo, ruptura de petróleo, tasa máxima, volumen poroso barrido, tiempo para ruptura de petróleo, tiempo para tasa máxima, tiempo de vida total del patrón, flujo fraccional de petróleo en el punto máximo, coeficiente de inyectividad, tasa de estado estable del patrón, tasa de petróleo en el punto máximo, saturación de agua en el banco, flujo fraccional de agua en el banco, espaciamiento en el patrón, área total desarrollada, número de patrones efectivos, volumen poroso inundable en el patrón, petróleo objetivo en el patrón, saturación de petróleo inicial, volumen poroso inundable en el proyecto, petróleo objetivo en el proyecto y recobro de petróleo total.

\section{Gráficas}

La interfaz de la herramienta permite presentar las variables de producción en gráficas con respecto al tiempo; igualmente, presenta la comparación de los resultados obtenidos de la predicción del proceso de inyección química con la predicción del proceso de inyección de agua. 


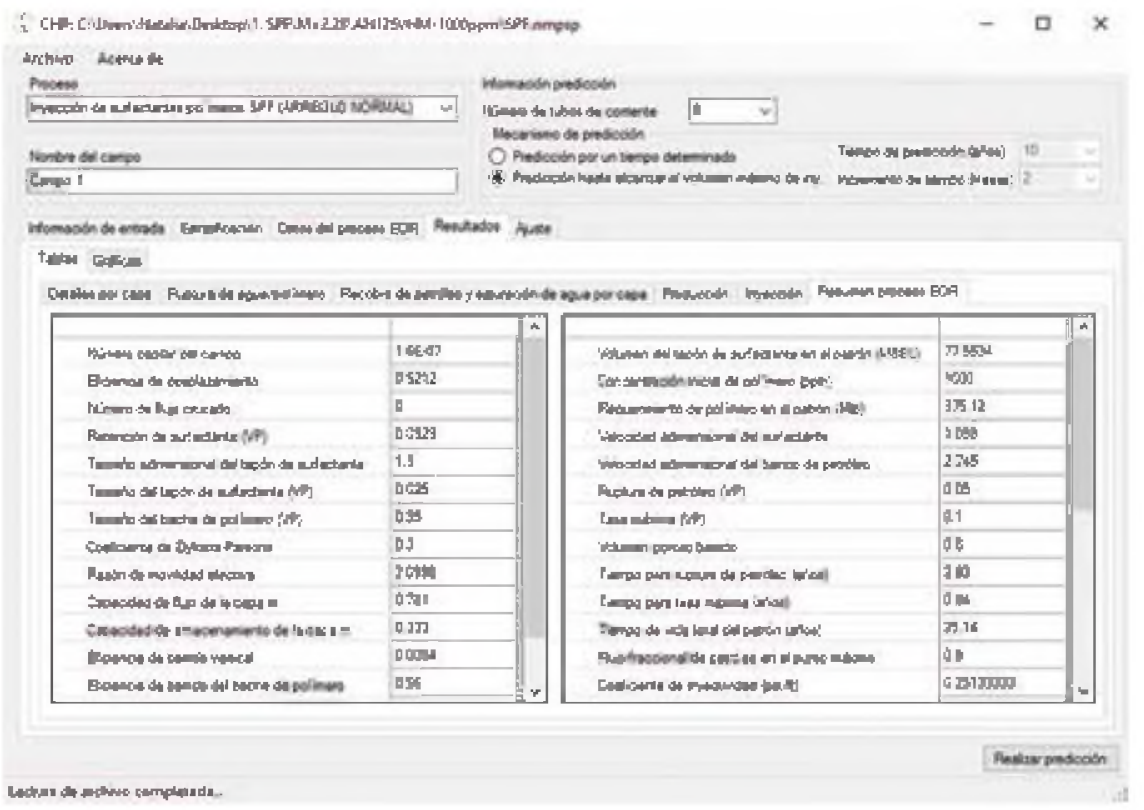

Figura 15. Ventana de resumen de variables utilizadas y resultantes en la predicción de procesos de inyección química. Aplicación CHP desarrollada por los autores.

\section{Producción de petróleo y agua}

Los resultados de producción se presentan como se ve en la figura 16 y se comparan con la tasa de inyección de agua.

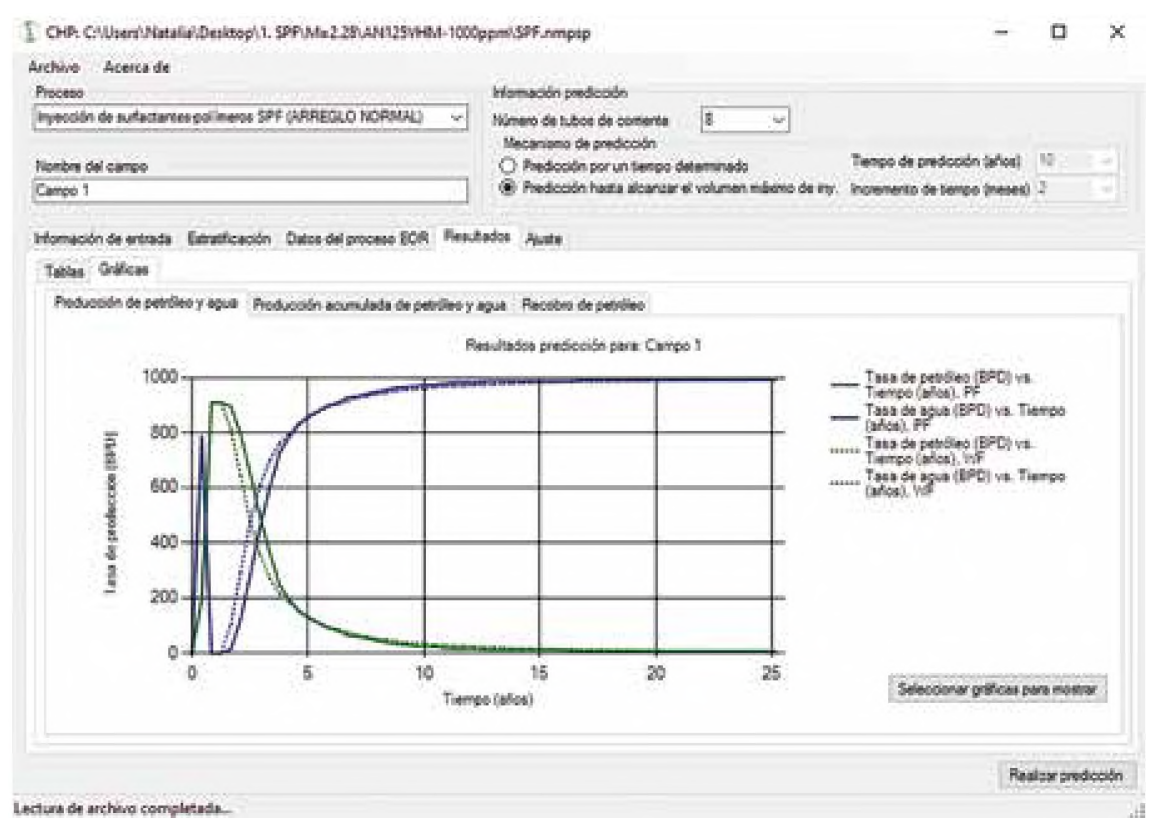

Figura 16. Ventana de resultados de producción diaria de petróleo y agua con respecto al tiempo. Aplicación CHP desarrollada por los autores. 


\section{Producción acumulada de petróleo y agua}

La producción acumulada de fluidos se presenta tal como se observa en la figura 17.

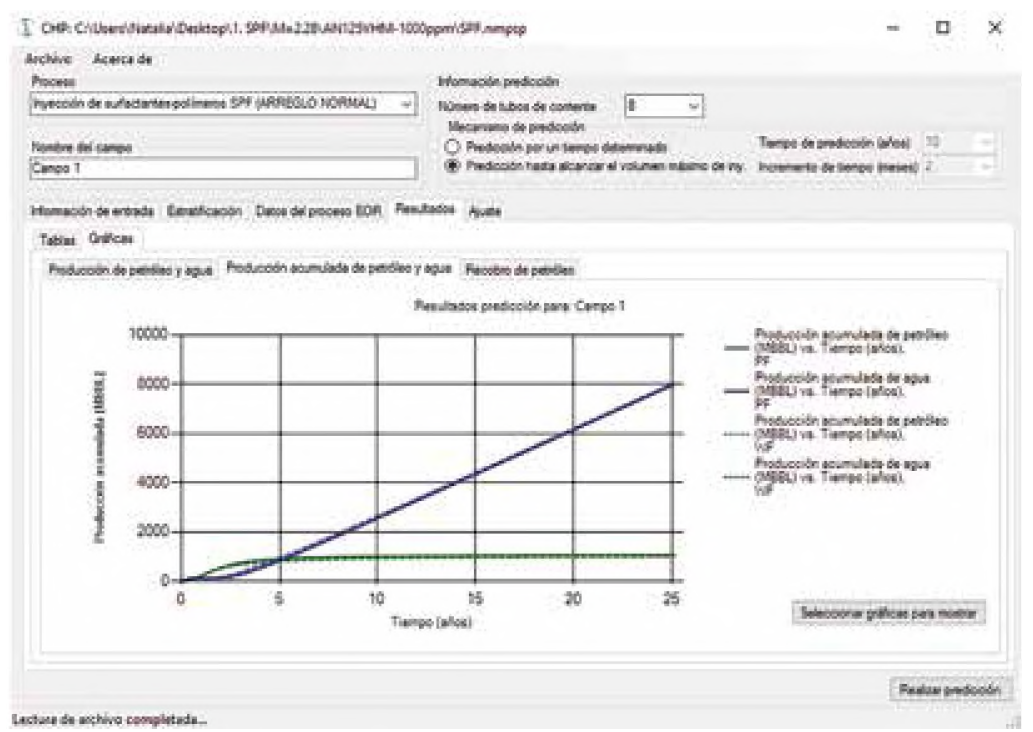

Figura 17. Ventana de resultados de producción acumulada de petróleo y agua con respecto al tiempo. Aplicación CHP desarrollada por los autores.

\section{Recobro de petróleo}

El factor de recobro de petróleo aparece junto con el volumen poroso inyectado, tal como se aprecia en la figura 18.

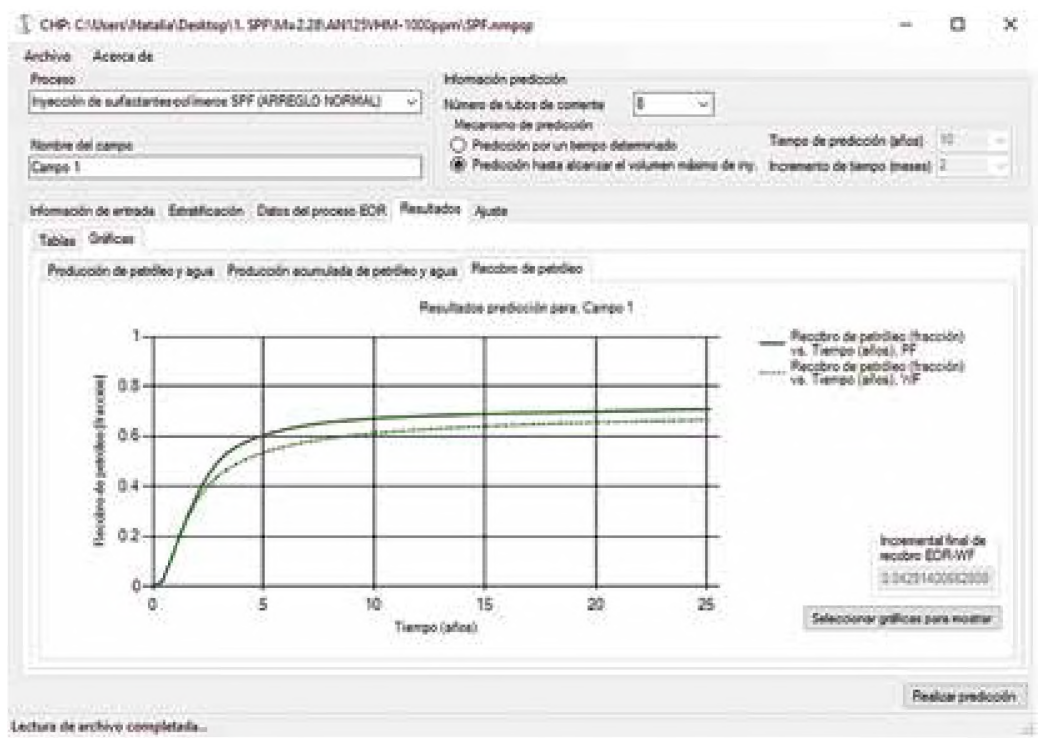

Figura 18. Ventana de resultados de factor de recobro de petróleo con respecto al tiempo. Aplicación CHP desarrollada por los autores. 


\section{AJuste}

Por último, se pueden comparar los resultados obtenidos por medio de la aplicación CHP con los resultados de un modelo diferente. Para este fin se debe importar un documento de texto que incluya los siguientes datos (ver el formato que se muestra en la figura 19): tiempo, tasa de petróleo, tasa de agua, petróleo acumulado, agua acumulada y factor de recobro, los cuales son organizados por la aplicación en una tabla (ver figura 20).

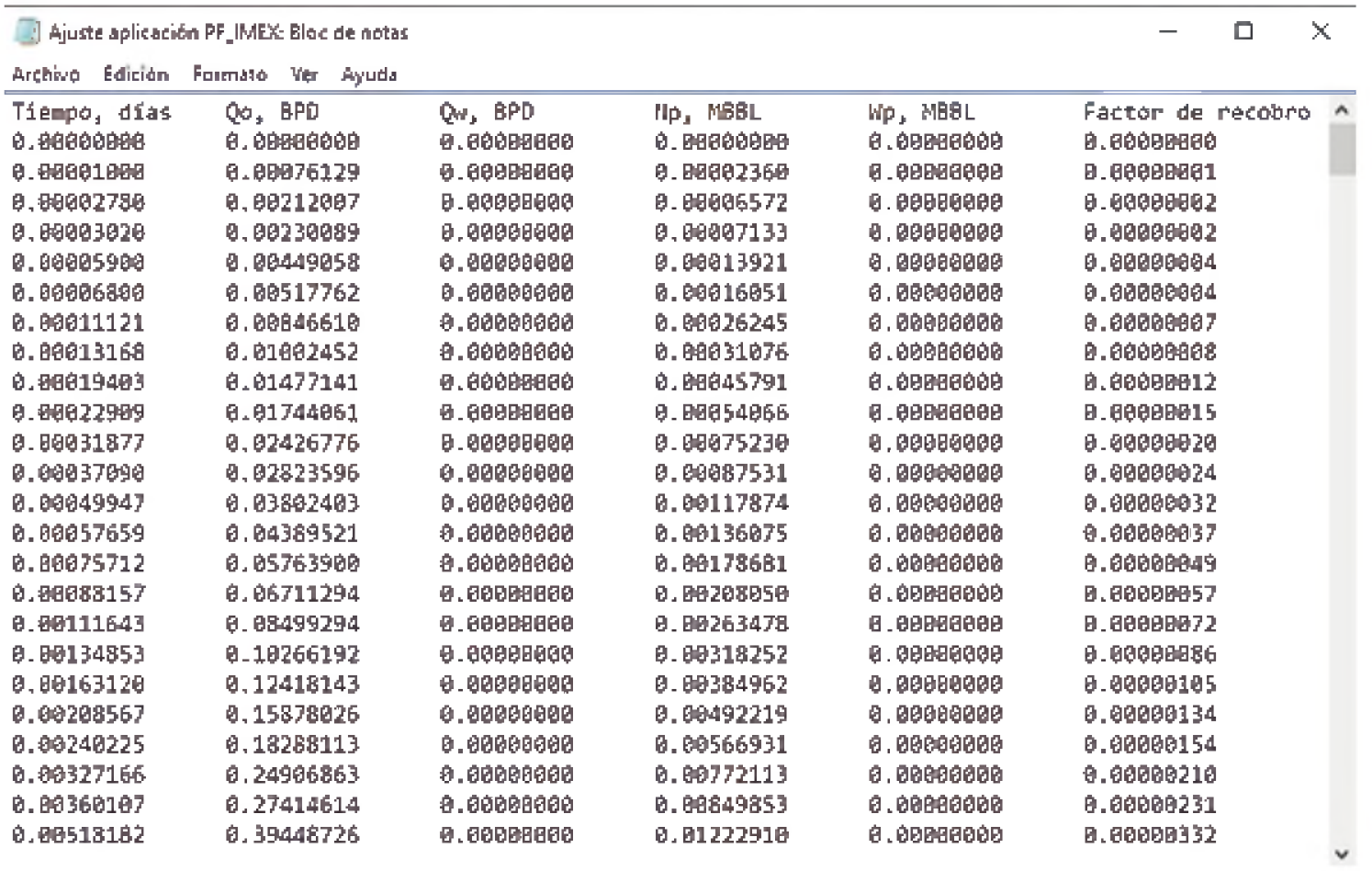

Figura 19. Archivo de texto para importar en la aplicación CHP. Aplicación CHP desarrollada por los autores.

A partir de la información anterior, se generan las gráficas que permiten visualizar los resultados de dos métodos de predicción del desempeño de un yacimiento bajo cierto esquema de inyección. La interfaz es muy similar a la de resultados; en este caso, la línea punteada representa los resultados de la herramienta predictiva CHP, y la línea sólida, los resultados del otro método escogido.

De esta manera se obtienen gráficas para comparar resultados de tasas de producción diaria (figura 21), producción acumulada (figura 22) y factor de recobro (figura 23), con respecto al tiempo. 


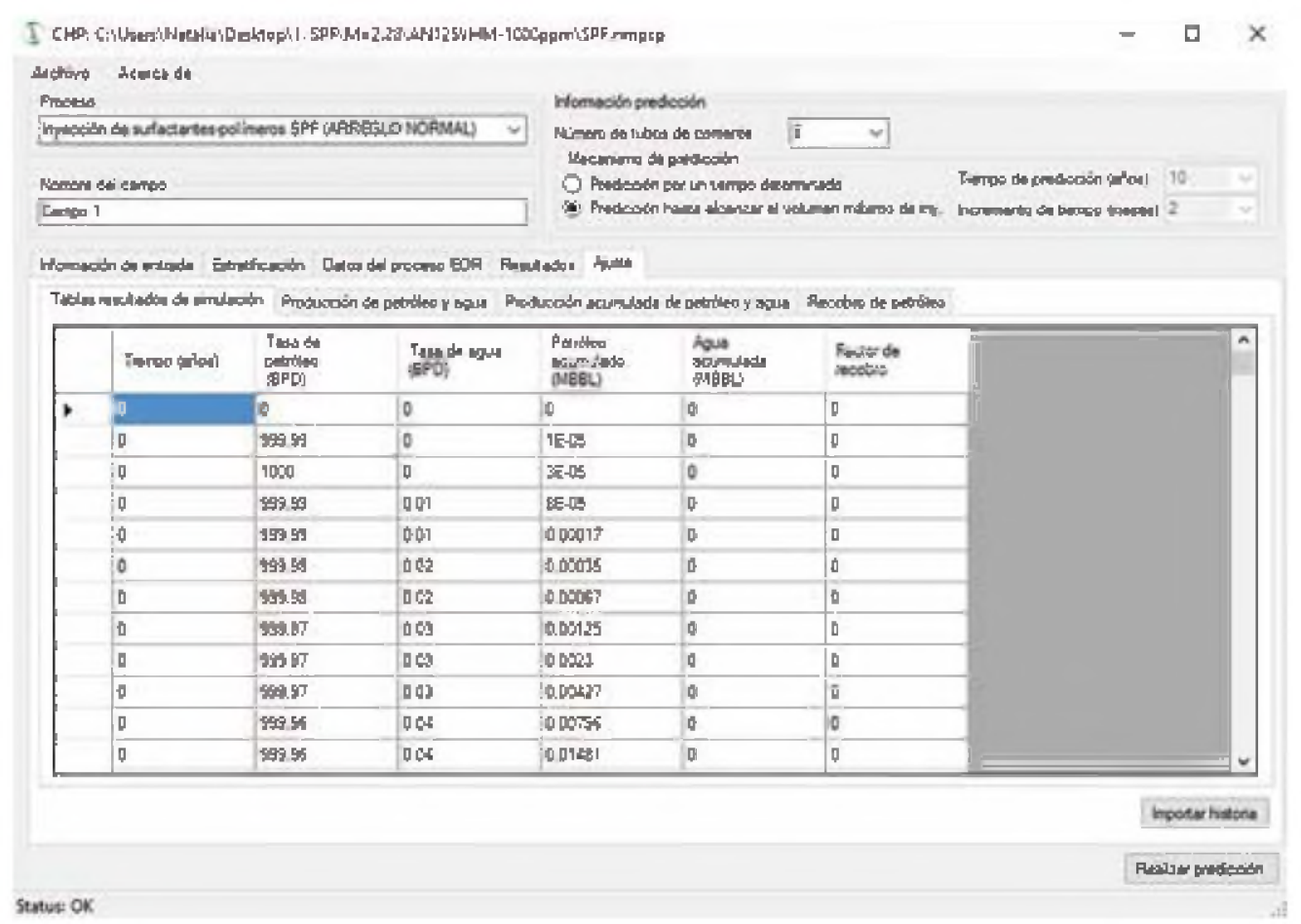

Figura 20. Ventana de los datos importados en la aplicación. Aplicación CHP desarrollada por los autores.

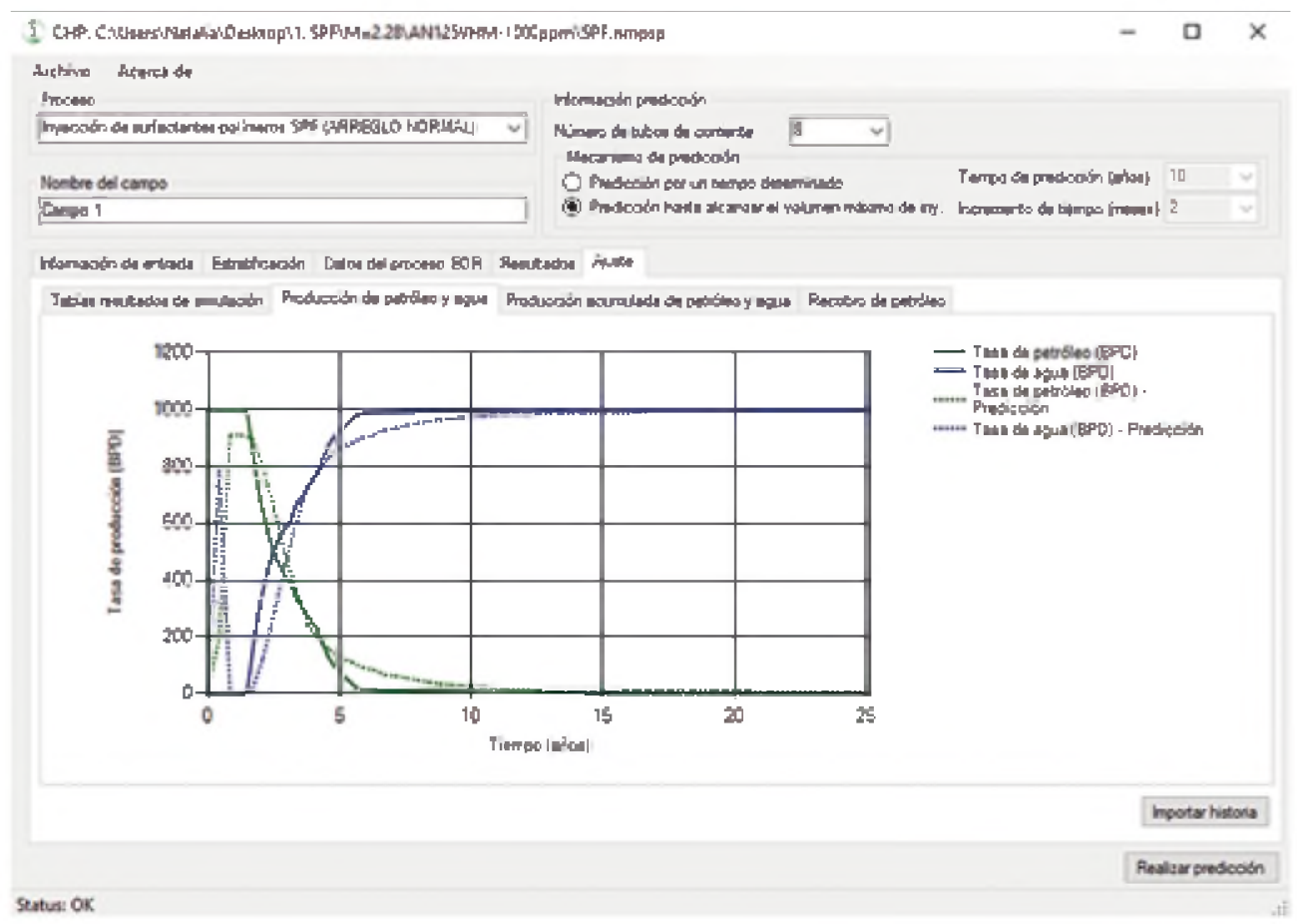

Figura 21. Ventana de comparación de producción diaria de petróleo y agua con respecto al tiempo. Aplicación CHP desarrollada por los autores. 


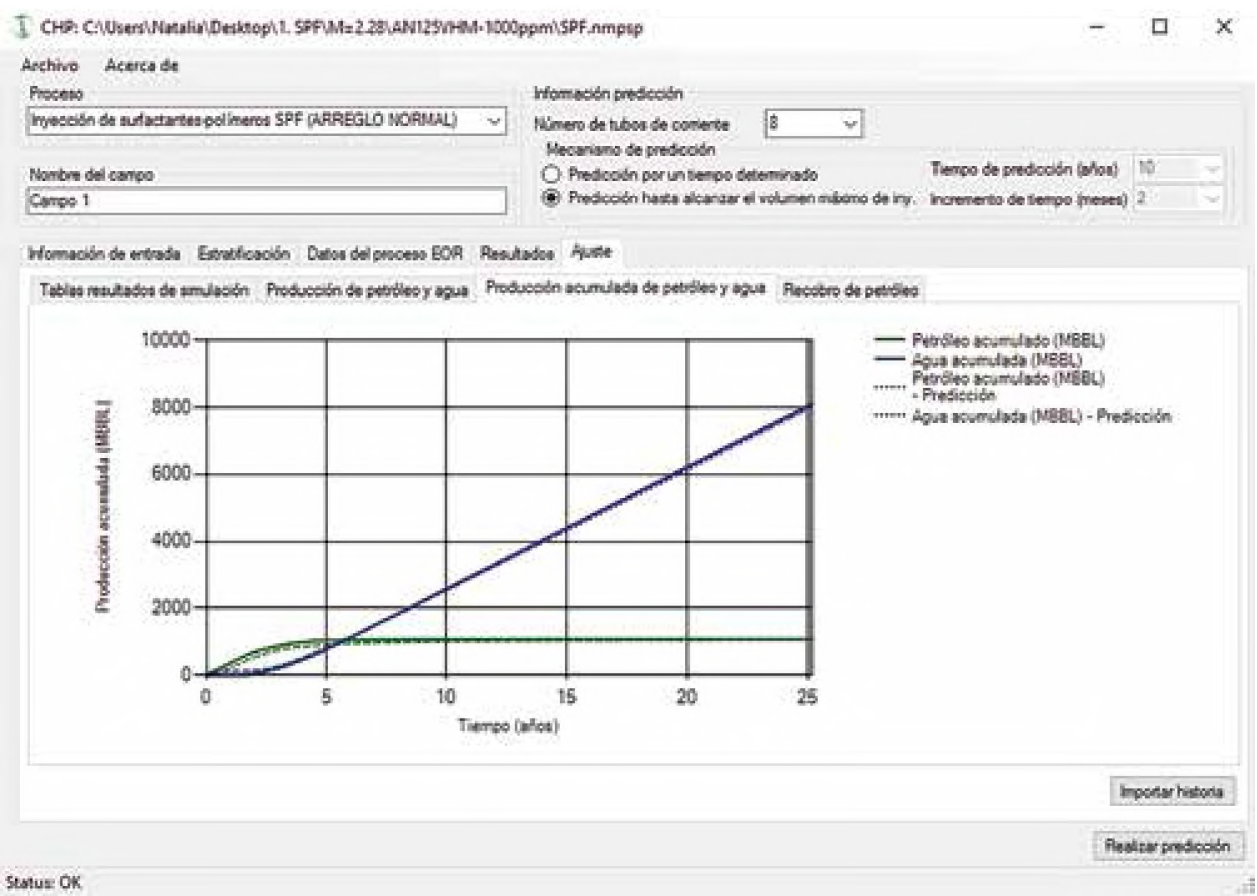

Figura 22. Ventana de comparación de producción acumulada de petróleo y agua con respecto al tiempo. Aplicación CHP desarrollada por los autores.

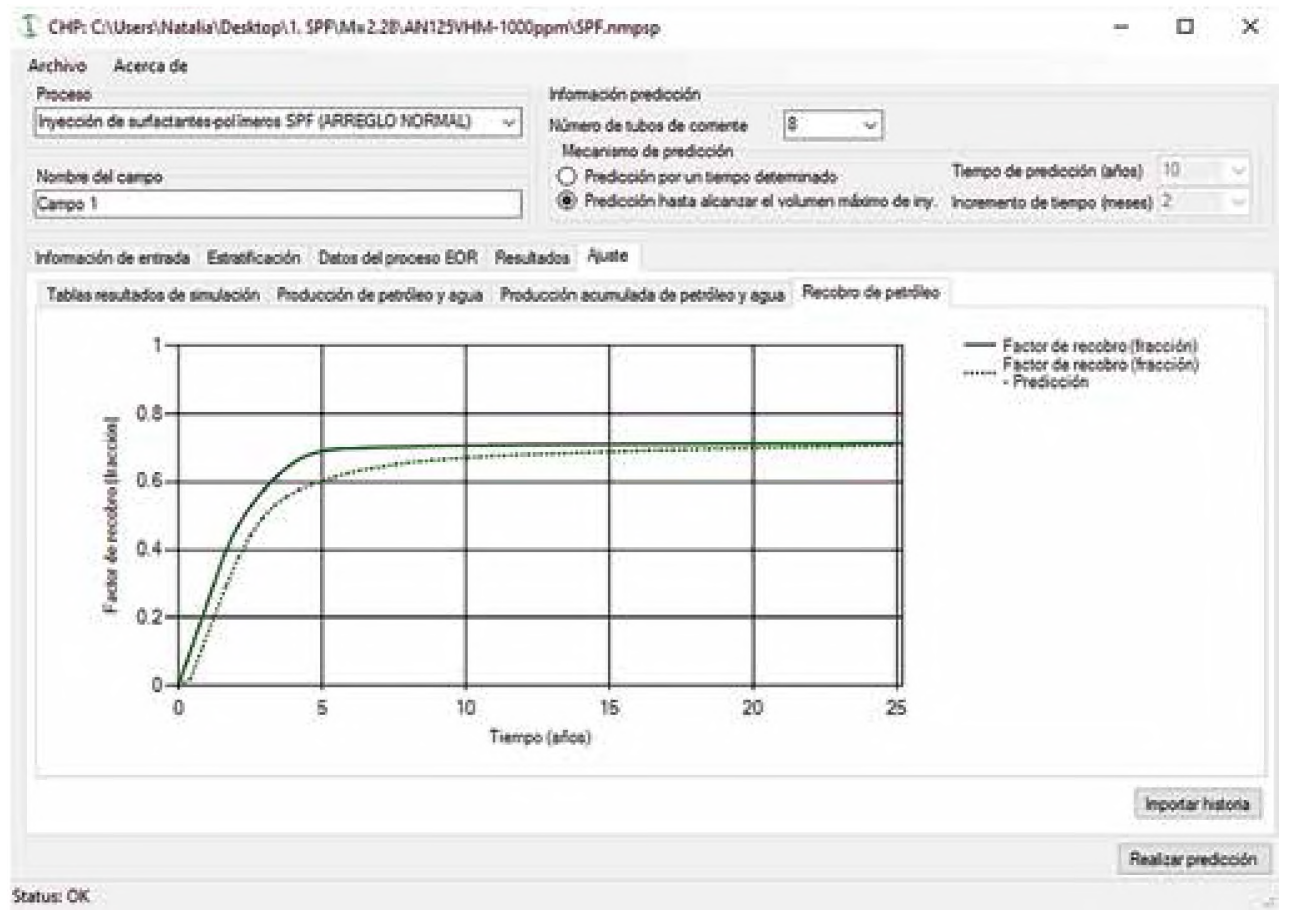

Figura 23. Ventana de comparación de factor de recobro de petróleo con respecto al tiempo. Aplicación CHP desarrollada por los autores. 


\section{Conclusiones}

La aplicación CHP es capaz de reproducir con poca información el comportamiento de recuperación de petróleo de un yacimiento bajo un esquema de inyección de agua, polímeros, surfactantes o surfactantes-polímeros; además, permite comparar los resultados obtenidos con los resultados de otros modelos. De acuerdo al estudio que se llevó a cabo por los autores y según se verificó con el desarrollo de los modelos predictivos y la validación con respecto al simulador CMG, se concluye que la aplicación realiza pronósticos con porcentajes de error inferiores al 11\%.

La estructura de la aplicación tiene una interfaz gráfica sencilla, de fácil manejo y entendimiento, integrada por un conjunto de módulos que realizan cálculos internos para las propiedades de los fluidos, la roca, los químicos inyectados, entre otros, y minimizan la cantidad de información requerida al usuario para la predicción del modelo.

\section{RefEREnCias}

Ahmed, T. (2006). Reservoir engineering handbook (3rd ed.). United States of America: Gulf Professional Publishing.

Angarita, N., Buitrago, S., y Ramos, J. (2016). Desarrollo de un modelo predictivo para inyección de quimicos surfactantes-polimeros convencional (tesis de pregrado). Facultad de Ingeniería, Fundación Universidad de América, Bogotá, Colombia.

Department of Energy of the United States of America y Ministry of Energy and Mines of the Republic of Venezuela. (1986a). Chemical flood predictive model, Supporting technology for enhanced oil recovery, fossil energy report III-5. EE. UU.: Department of Energy of the United States of America.

Department of Energy of the United States of America y Ministry of Energy and Mines of the Republic of Venezuela. (1986b). Polymer predictive model, Supporting technology for enhanced oil recovery, fossil energy report III-4. EE. UU.: Department of Energy of the United States of America.

Honarpour, M., Koederitz, L., y Harvey, H. (1986). Relative permeability of petroleum reservoirs. Boca Raton, Florida: CRC Press Inc.

Paris de Ferrer, M. (2001). Inyección de agua y gas en yacimientos petrolíferos. Maracaibo, Venezuela: Astro Data S. A.

Petroleum Solutions Ltd. (2010). EORgui Help [manual de ayuda Software EOR]. EE. UU.: Petroleum Solutions Ltd.

Willhite, G. (1986). Waterflooding ( $3^{\mathrm{a}}$ ed.). Texas: Society of Petroleum Engineers. 
